\title{
Rate transient analysis of Well-07 and Well-10 of Habiganj gas field, Bangladesh
}

\author{
Mir Raisul Islam ${ }^{1}$ (1) • A. T. M. Shahidul Huqe Muzemder ${ }^{1}$ • \\ Md. Ashraful Islam Khan ${ }^{1}$ Md. Mahbub Alam Hira ${ }^{1}$
}

Received: 11 February 2016/ Accepted: 31 July 2016/Published online: 13 August 2016

(c) The Author(s) 2016. This article is published with open access at Springerlink.com

\begin{abstract}
Rate transient analysis (RTA) plays a significant role in the research and development sector of petroleum industry to know about the reserves and physical properties of commercially expected hydrocarbon fluids of a petroleum field. In this research, to conduct a rate transient analysis study; reservoir properties, well properties and Production data over the year of 2007 from two gas producing wells, Well-07 and Well-10 of Habiganj gas field, Bangladesh were used. There are two gas zones in this gas field: upper gas sand (UGS) and lower gas sand (LGS). Only UGS was considered as a gas reservoir in this research work due to no producing well in LGS. Software FEKETE, F.A.S.T.RTA ${ }^{\mathrm{TM}}$ (version 4.5.1.277), IHS Inc. was used to conduct this research. The objectives of this research were to estimate gas initially in place (GIIP) and expected ultimate recovery (EUR) of Well-07 and Well-10, to determine permeability and skin surrounding each of these two producing wells. After completion of the analysis, the GIIP and EUR values of Well-07 were estimated to 435.082 billion cubic feet (Bcf) and $304.558 \mathrm{Bcf}$, and those of 475.242 Bcf and 332.67 Bcf of Well-10, respectively. Skin effect and permeability in the surrounding of each of these producing wells were amounted to 7.017 and 3.0396 millidarcy (md) for Well-07 and those of 7.014 and $2.7839 \mathrm{md}$ for Well-10, respectively, by the end of the year of 2007 .
\end{abstract}

Mir Raisul Islam

rockypme@gmail.com

1 Department of Petroleum and Mining Engineering, Shahjalal University of Science and Technology, Sylhet 3114, Bangladesh

(2007.

Keywords Rate transient analysis · Habiganj gas field . Typecurve analysis · Decline curve analysis · Gas initially in place $\cdot$ Expected ultimate recovery $\cdot$ Skin $\cdot$ Permeability

\section{List of symbols}

F $\quad$ Fahrenheit

$S \quad$ Skin factor

$S_{\mathrm{d}} \quad$ Skin effect due to well drilling and completion

$S_{\mathrm{PT}} \quad$ Pseudo-skin factor resulted from reservoir open level

$S_{\mathrm{PF}} \quad$ Pseudo-skin factor due to perforation

$r_{\mathrm{s}} \quad$ Radius of the altered zone due to skin

$k_{\mathrm{s}} \quad$ Permeability of the altered zone due to skin

$\mathrm{k} \quad$ Permeability of the reservoir (md)

$r_{\mathrm{w}} \quad$ Wellbore radius

$r_{\mathrm{e}} \quad$ Radius of the reservoir

$\Delta p_{\mathrm{s}} \quad$ Additional pressure drop due to skin effect

$\mu \quad$ Viscosity

$q \quad$ Production rate (MMScfd)

$\Delta p_{\mathrm{p}} \quad$ Pseudo-pressure difference (psi)

$P_{\text {pwf }} \quad$ Bottomhole pseudo-pressure (psi)

$Q=G \quad$ Cumulative production (Bcf)

$Q_{\mathrm{G}} \quad$ Cumulative gas production (Bcf)

$t_{c} \quad$ Material-balance time

$\mathrm{t}_{\mathrm{ca}} \quad$ Material-balance pseudo-time

$\mu_{\mathrm{g}} \quad$ Viscosity of gas

$c_{\mathrm{g}} \quad$ Compressibility of gas $\left(\mathrm{psi}^{-1}\right)$

$q_{\mathrm{g}} \quad$ Gas production rate (mmcfd)

$t \quad$ Time (day)

$r_{\text {ed }} \quad$ Dimensionless radius of the reservoir

$t_{\mathrm{Dd}} \quad$ Dimensionless time

$q_{\text {Dd }} \quad$ Dimensionless rate

$h \quad$ Net pay thickness of the reservoir (ft)

$C_{\mathrm{t}} \quad$ Total compressibility $\left(\mathrm{psi}^{-1}\right)$

$P_{\mathrm{i}} \quad$ Initial pressure (psi)

$P_{\text {i }}$ 
$P_{\mathrm{pi}} \quad$ Initial pseudo-pressure (psi)

$P_{\mathrm{wf}} \quad$ Wellbore flowing pressure (psi)

$P_{\text {pwf }} \quad$ Wellbore flowing pseudo-pressure (psi)

$t_{\mathrm{dA}} \quad$ Dimemsionless radius

A Area $\left(\mathrm{ft}^{2}\right)$

$B \quad$ Formation volume factor

\section{Abbreviations}

A-G Agarwal-Gardner

Bcf Billion cubic feet

BGFCL Bangladesh Gas Field Company Limited

DCA Decline curve analysis

EUR Expected ultimate recovery

FWHP Flowing well head pressure

GIIP Gas initially in place/gas initial in place

GWC Gas water contact

Inc. Incorporation

IKM Intercomp-Kanata Management Limited

$\mathrm{km} \quad$ Kilo meter

LGS Lower gas sand

md Millidarcy

NPI Normalized pressure integral

PDA Production data analysis

PTA Pressure transient analysis

RTA Rate transient analysis

SPE Society of Petroleum Engineers

Tcf Trillion cubic feet

UGS Upper gas sand

Well-07 Production Well no. 07 drilled in chronological order

Well-10 Production Well no. 10 drilled in chronological order

\section{Introduction}

Habiganj gas field, Bangladesh, was the study area to carry out this research. This gas field is located at Madhabpur Upazila, Habiganj District, about 100 kilo meter $(\mathrm{km})$ away to the direction of northeastern from Dhaka (capital city of Bangladesh). This gas field lies about $32 \mathrm{~km}$ northeast of Titas gas field, the largest reserved gas field in Bangladesh. This gas field was discovered by shell Oil Company in 1963 and has been one of the major gas producers in Bangladesh (Imam 2013; Bangladesh Gas Fields Company Limited [BGFCL] 2014). Figure 1 shows the location of Habiganj gas field in Habiganj District in Bangladesh. It is the third-largest gas producer in the country and operated by Bangladesh Gas Field Company Ltd (BGFCL), a subsidiary of Bangladesh Oil, Gas and Mineral Corporation (known as Petrobangla) (Imam 2005).

There are two gas zones, upper gas sand (UGS) and lower gas sand (LGS). These two zones are sandstone formations. The UGS is the primary gas reservoir of the gas field and lies at a depth of $1320 \mathrm{~m}$ below the surface. It has a maximum gross pay of $230 \mathrm{~m}$ thick. The gas sand is medium to fine grained, well sorted, clean and unconsolidated. It has an average porosity of $30 \%$ and average permeability in the range of 2-4 darcy (Imam 2013). Figure 2 represents the cross section of the subsurface of Habiganj gas field, Bangladesh.

According to Haq and Gomes (2001), Intercomp-Kanata Management Limited (IKM) Reservoir Engineering Report (1990) stated that the recovery from the UGS in the Habiganj gas field is dominated by the water drive mechanism and the aquifer to be ten times extensive than the reservoir. Hartmann and Beaumont (2016) says that water drive mechanism exists only where the aquifer is of equal or better quality and has a much larger volume than the reservoir (about 10 times).

\section{Rate transient analysis}

Rate transient analysis (RTA) is the science of analyzing production data (both rates and flowing pressures). This method is an important tool to estimate reserve of oil and/or gas of a reservoir. Reserve estimation and development planning are the key tasks of petroleum engineers by the use of historical production (reservoir fluid production rate histories and cumulative production). Both of these fall within the domain of a quantitative production data analysis (PDA) (Help Manual 2010; Clarkson et al. 2012; Clarkson 2013).

RTA can also be defined as a modern decline curve analysis (DCA) method (Mishra 2014). DCA method is one of the oldest and most often used tools of the petroleum engineers. This is a forecasting technique which predicts by history matching of rate-time data on an appropriate typecurve. What direction to take, what typecurve(s) to choose and where the rate-time data should fit are decided based on basic reservoir engineering concepts and knowledge (Fetkovich 1980; Fetkovich et al. 1987). So DCA is not based on applying a purely empirical equation to be analyzed with statistical approaches which often leads to unrealistic and unreliable forecast and reserve estimate (Fetkovich et al. 1996).

RTA also gives convincing estimates of reservoir parameters with available low-frequency (weekly or monthly) production data. Once reservoir characteristics are determined using RTA, a reservoir model is then constructed to forecast future production scenarios (Mishra 2014; Mireault and Dean 2007-2008).

\section{Typecurve analysis}

According to Ley and Samaniego (1981), a considerable amount of information concerning well test analysis 


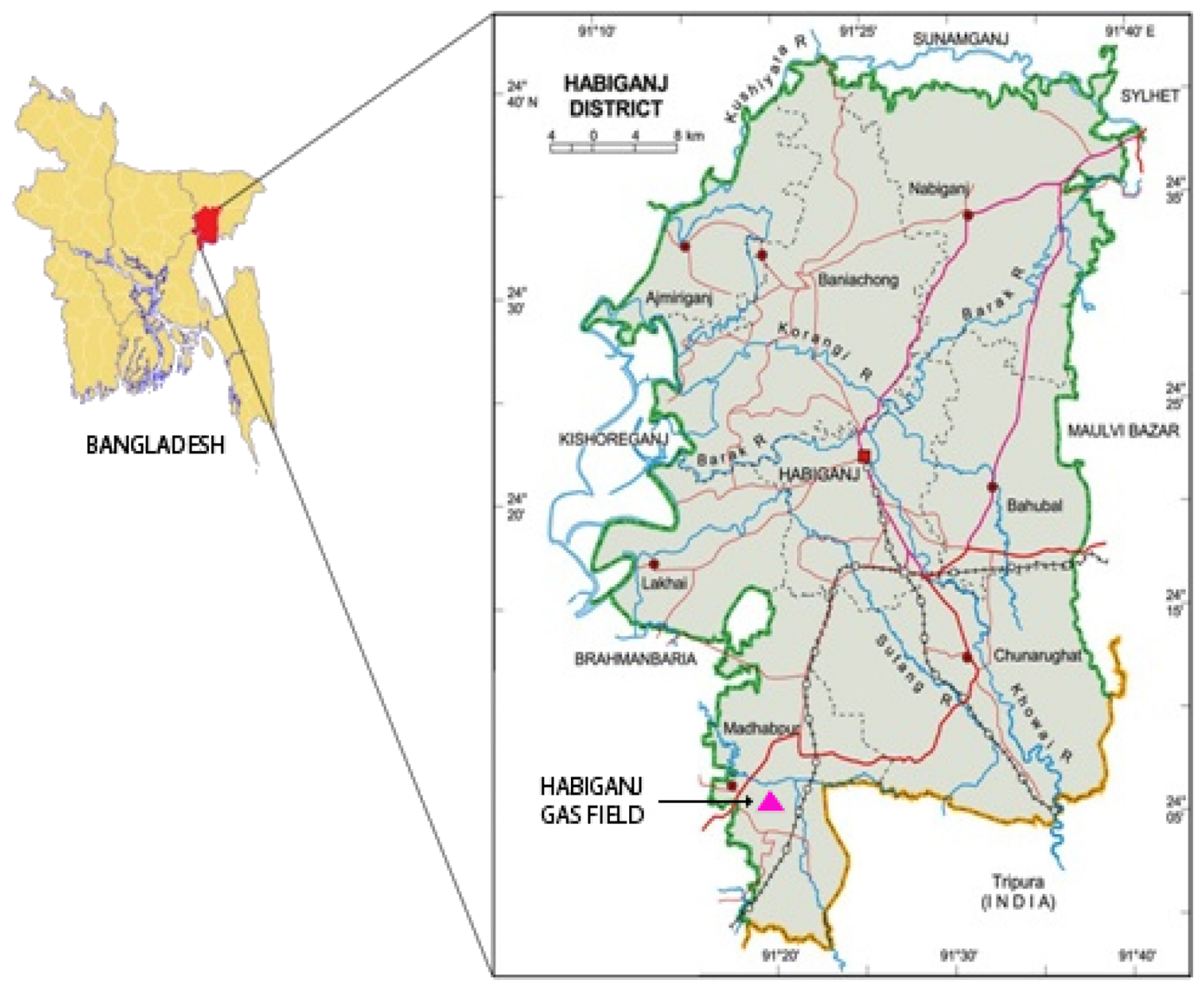

Fig. 1 Location of Habiganj gas field, Bangladesh Source: Wikipedia

has been in the literature over the last several decades. Typecurve analysis consists of finding a typecurve that matches the actual response of the well and the reservoir during the test. Then the reservoir and well parameters, such as permeability and skin, can be calculated from the dimensionless parameters defining that typecurve (Gringarten 1987).

\section{Gas initial in place and expected ultimate recovery}

Gas initial in place (GIIP) refers to the total amount of gas present initially in the underground of a gas field. Part of the GIIP in an explored gas field can be recovered. Generally, the recovery of gas from the GIIP in a typical gas field ranges from as low as $60 \%$ to as high as $90 \%$ (Imam 2013).
Expected ultimate recovery (EUR) of a petroleum source is the sum of the proven reserve at a specific time and the cumulative production up to that time. Proven reserve refers to the quantity of gas in a gas reservoir which can be estimated with reasonable certainty (high degree of confidence) to be commercially recoverable from known reservoir under the present economic and operating conditions (Imam 2013; Morehouse 1997).

\section{Permeability and skin}

Permeability $(k)$ in a reservoir rock is its capacity to transport fluids through a system of interconnected pores. Reservoir permeability is a random-valued property of the formation (Zolotukhin and Ursin 2000; Jensen et al. 1987). 

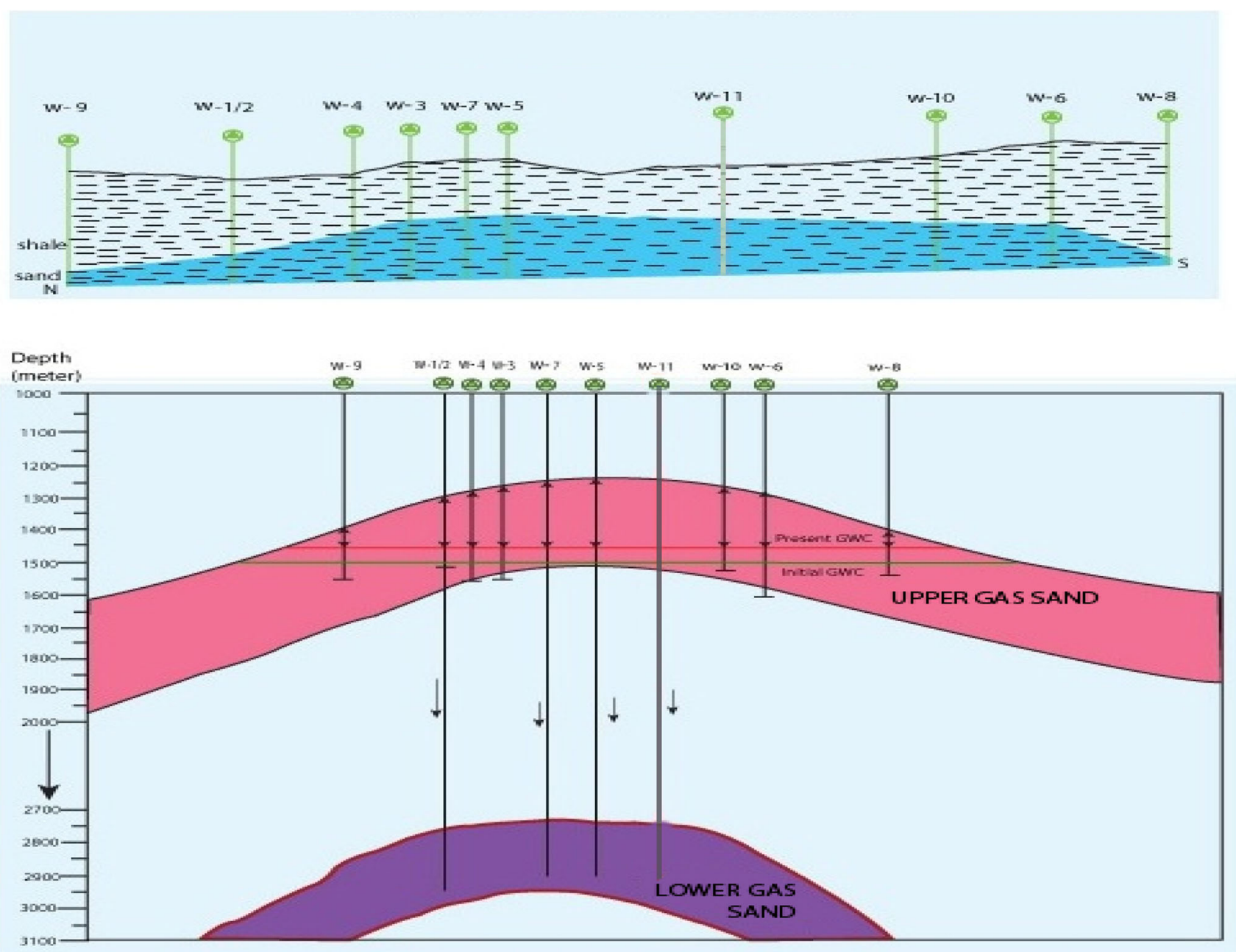

Fig. 2 Cross-sectional view of the subsurface of Habiganj gas field, Bangladesh (Imam 2013)

The pressure drop in a well per unit rate of flow is controlled by the resistance of the formation, the viscosity of the fluid, and the additional resistance concentrated around the wellbore due to drilling, completion and production practices. The pressure drop caused by this additional resistance is defined as the skin effect, denoted by the symbol S. The reservoir damage occurs because of this skin effect. Reservoir damage is resulted from well drilling and completion $\left(S_{\mathrm{d}}\right)$, pseudo-skin factor resulted from reservoir open level $\left(S_{\mathrm{PT}}\right)$ and pseudo-skin factor resulted from perforation $\left(S_{\mathrm{PF}}\right)$ and several other skin factors (Everdingen 1953; Jianchun et al. 2014).

A two-region reservoir model (Fig. 3) represents a damaged well. In this model, the altered zone around the wellbore is assumed to have uniform permeability $k_{\mathrm{s}}$ out to a radius $r_{\mathrm{s}}$, beyond which the formation permeability, $k$, is unaltered (Altered zone and skin effect 2016). Everdingen found that better agreement between theory and well performance is possible, if the permeability

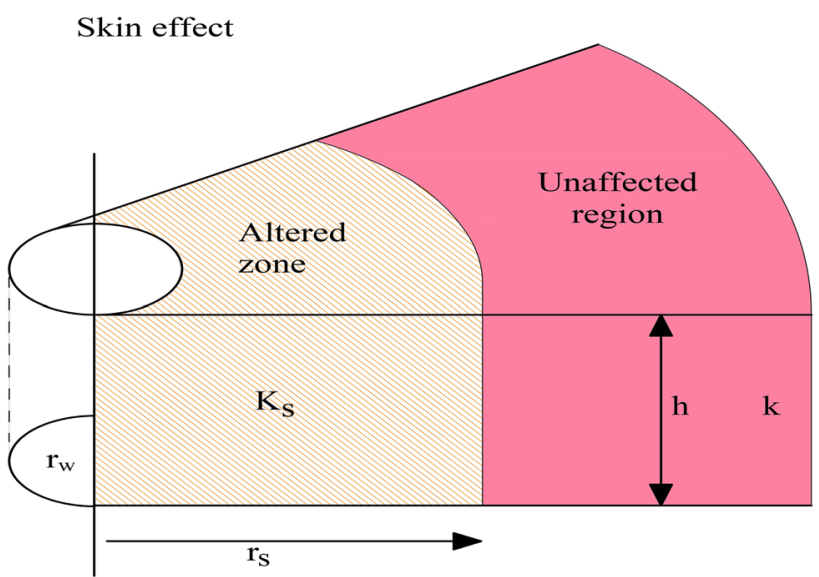

Fig. 3 Two-region reservoir model (Altered zone and skin effect 2016)

reduction near the wellbore region is assumed due to drilling, completion and production practices (Hawkins Jr. 1956). 


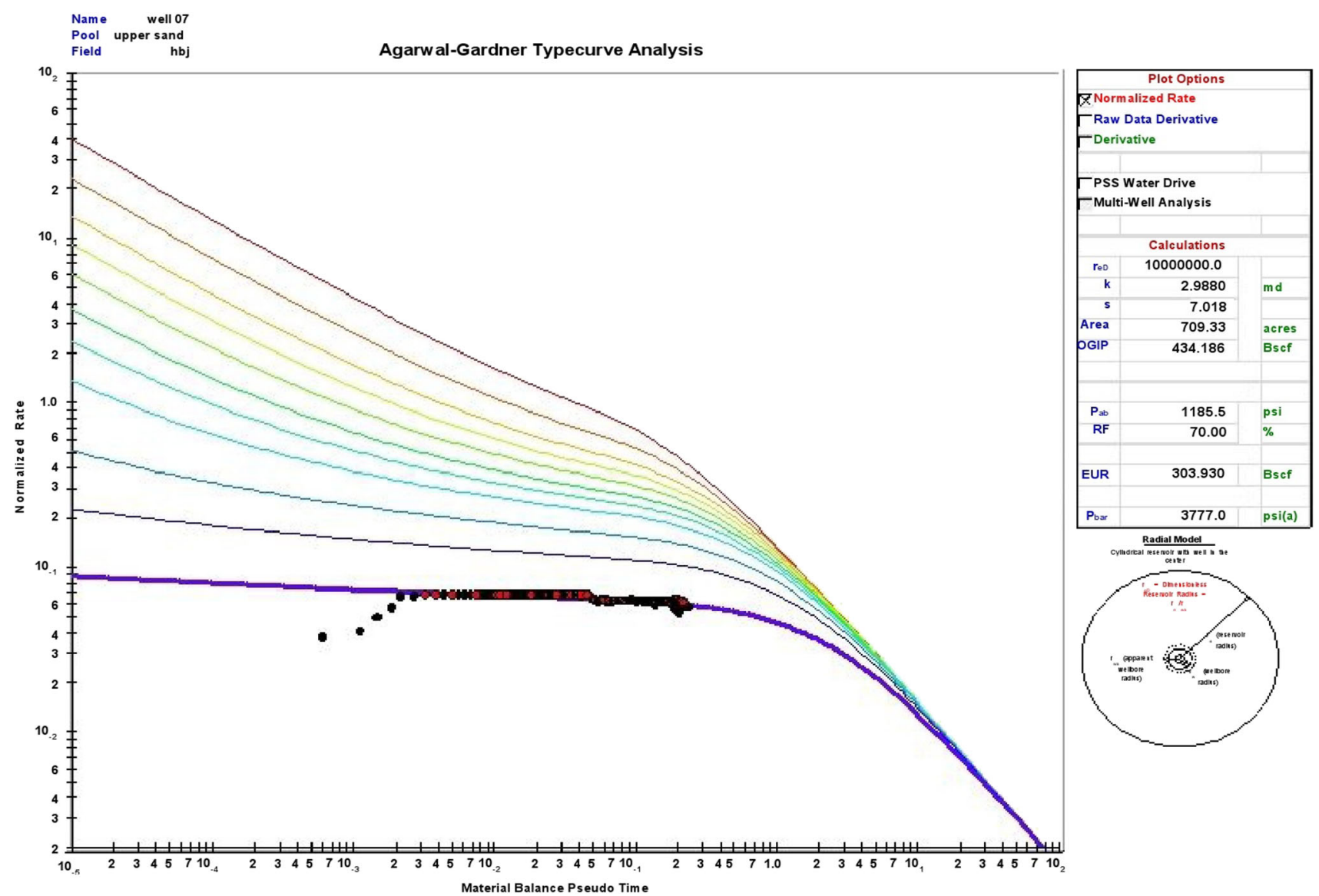

Fig. 4 Match between data plot for Well-07 and Agarwal-Gardner typecurve plot

\section{Materials and methods}

Data (reservoir properties, fluid properties, properties of Well-07 and Well-10, and production data of Well-07 and Well-10 for the year of 2007) were collected from BGFCL, a subsidiary of Petrobangla for this study. By using software FEKETE, F.A.S.T.RTA ${ }^{\mathrm{TM}}$, methods such as Blasingame typecurve analysis, Agarwal-Gardner typecurve analysis and normalized pressure integral (NPI) typecurve analysis were followed. After entering data into the software, data points in the graph were seemed to be very scattered. It was essential to be filtered. To minimize error and to obtain a good match between typecurves and data plot, data points in the graph of the software were filtered to clean up "noise" and to reduce data which were scattered.

The major assumptions in this work were as follows:

1 The reservoir is assumed to be homogeneous.

2 The reservoir is circular of radius $r_{\mathrm{e}}$.

3 The fluid is of constant viscosity, $\mu$.

4 Radial flow of fluids.

\section{Required data for the study}

The required reservoir, fluid and well properties are numerated below. A table concerning the report of daily flowing well head pressure (FWHP) and daily production of gas and water for the year of 2007 are presented in Appendices 1 and 2 section in this paper.

\section{Reservoir properties}

Producing zone: upper gas sand

Porosity: $30 \%$

Reservoir temp: 178 degree F

\section{Fluid properties}

Fluid type: gas

Fluid used for static calculation: gas 

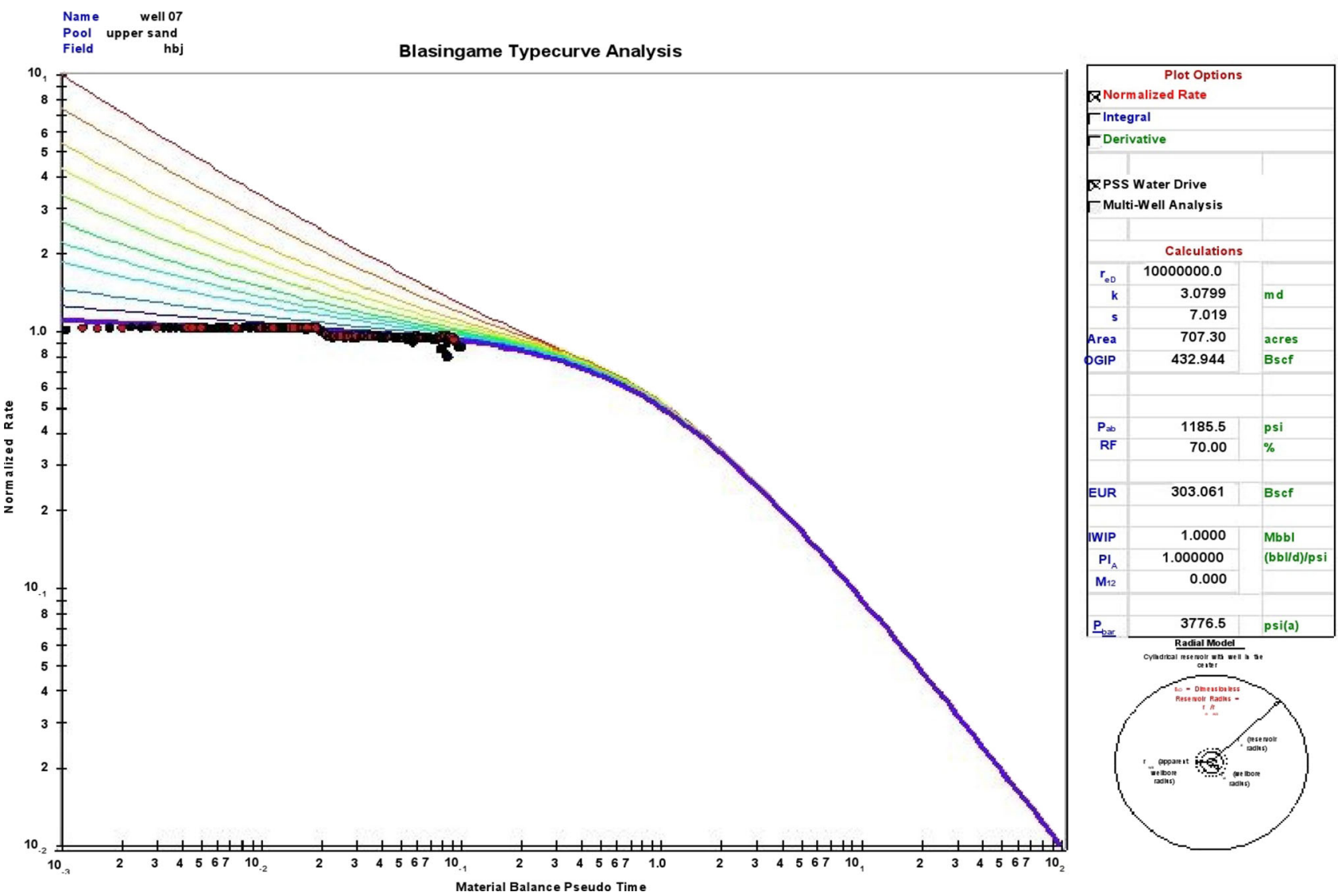

Fig. 5 Match between data plot for Well-07 and Blasingame typecurve plot

\section{Properties of Well-07}

Well type: vertical

Perforation interval: $4347-4600 \mathrm{ft}$

Midpoint perforation: $4473.5 \mathrm{ft}$

Tubing size: 4.5 in

$R_{\mathrm{w}}: 0.350 \mathrm{ft}$

Casing (ID): 7 in

Static Well head temp: 75 degree F (avg)

Flowing Well head temperature: 95 degree F (avg)

\section{Properties of Well-10}

Well type: vertical

Perforation interval: 4373-4649 ft

Midpoint perforation: $4511 \mathrm{ft}$

Tubing size: 4.5 in

$R_{\mathrm{w}}: 0.350 \mathrm{ft}$

Porosity: $30 \%$

Casing (ID): 7 in

Static Well head temp: 75 degree F (avg)

Flowing Well head temperature: 95 degree F (avg)

\section{Blasingame typecurve analysis}

At Blasingame method (Help Manual 2010), the normalized rate was plotted against material-balance pseudo-time on a $\log -\log$ scale of the same size as the typecurves, which are referred as the "data plot".

For data plot, normalized rate, $\frac{q}{\Delta \mathrm{p}_{\mathrm{p}}}=\frac{q}{\left(p_{i}-p_{\mathrm{pwf}}\right)}$

Material balance time $t_{c}=\frac{Q}{q}$

Application of this concept to oil would be very straight forward. To gas, it is more complex because of varying PVT properties of gas. Accordingly simple concept $t_{c}=\frac{G_{g}}{q_{g}}$ must be defined in terms of pseudo-time,

$t_{\mathrm{ca}}=\left\{\frac{\left(\mu_{\mathrm{g}} c_{\mathrm{g}}\right)_{j}}{q_{\mathrm{g}}}\right\} \int_{o}^{t}\left\{\frac{q_{\mathrm{g}}}{\mu_{\mathrm{gav}} c_{\mathrm{gav}}}\right\} \mathrm{d} t$

Blasingame et al. established his typecurves using dimensionless rate $\left(q_{\mathrm{Dd}}\right)$ against dimensionless time $\left(t_{\mathrm{Dd}}\right)$ on a $\log -\log$ scale. 


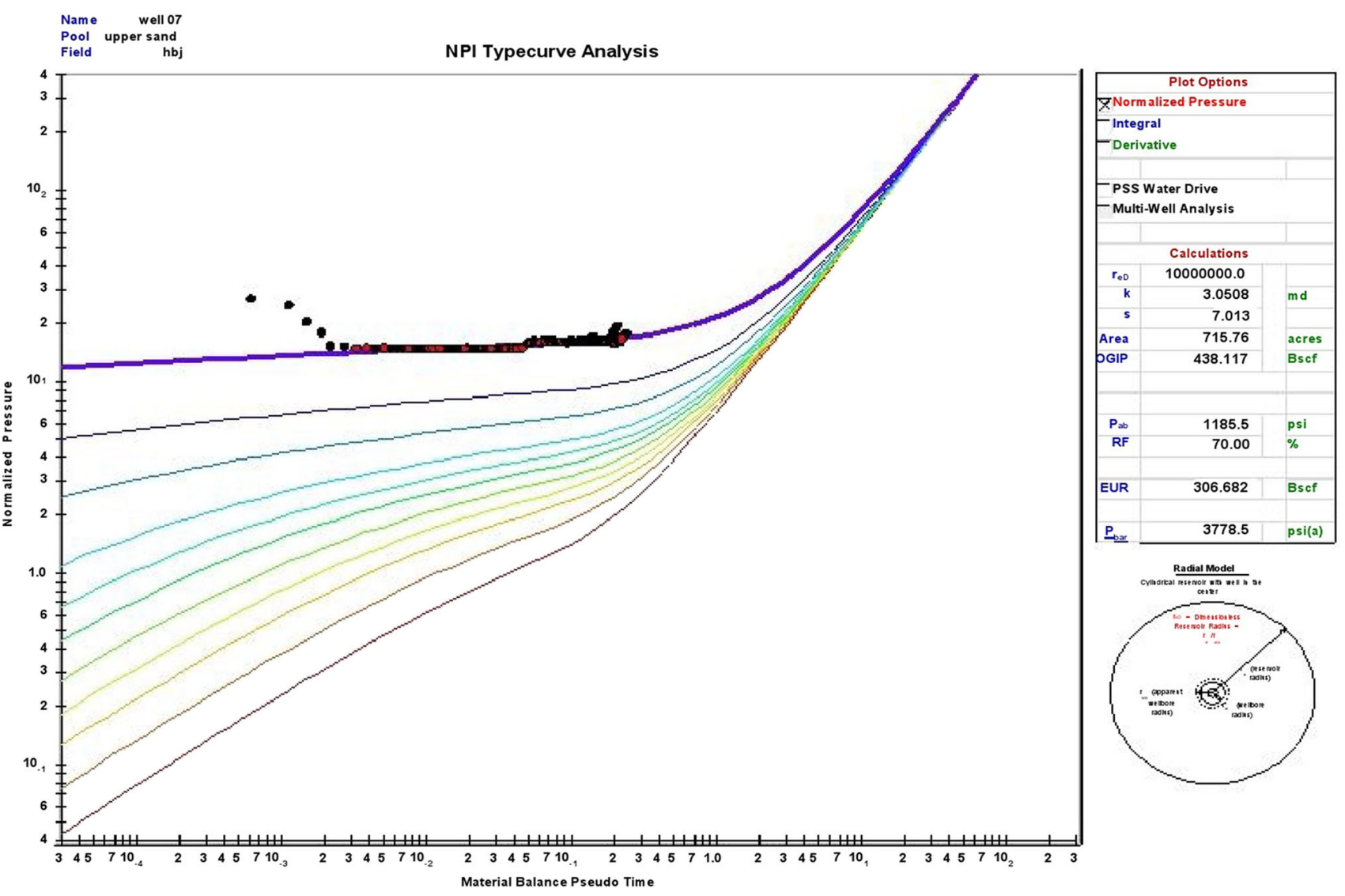

Fig. 6 Match between data plot for Well-07 and NPI typecurve plot

$$
\begin{gathered}
q_{\mathrm{Dd}}=q_{\mathrm{d}} \ln \left(r_{\mathrm{eD}}-0.5\right), r_{\mathrm{eD}}=\frac{r_{\mathrm{e}}}{r_{\mathrm{w}}} \\
t_{\mathrm{Dd}}=\frac{2 t_{d}}{\left.\left\{\left(\ln r_{\mathrm{eD}}\right)-0.5\right)\left(r_{\mathrm{eD}}^{2}-1\right)\right\}}
\end{gathered}
$$

\section{Agarwal-Gardner typecurve analysis}

At Agarwal-Gardner method (Help Manual 2010), the procedure is almost similar to the Blasingame with a few dissimilarities. As opposed to Blasingame, here for data plot,

Normalized rate, $\frac{q}{\Delta p_{\mathrm{p}}}=\frac{q}{\left(p_{\mathrm{i}}-p_{\mathrm{pwf}}\right)}$

Material balance pseudo time, $t_{\mathrm{ca}}$

$$
=\left\{\frac{\left(\mu_{\mathrm{g}} c_{\mathrm{g}}\right)_{j}}{q_{\mathrm{g}}}\right\} \int_{o}^{t}\left\{\frac{q_{\mathrm{g}}}{\mu_{\mathrm{gav}} c_{\mathrm{gav}}}\right\} \mathrm{d} t
$$

Agarwal et al. established his typecurves using dimensionless rate $\left(q_{\text {Dd }}\right)$ against dimensionless time $\left(t_{\mathrm{Dd}}\right)$ on a $\log -\log$ scale as,

$$
\begin{aligned}
q_{\mathrm{D}} & =\frac{1}{p_{\mathrm{D}}}=141.2 \frac{q B \mu}{\left\{k h\left(p_{\mathrm{i}}-p_{\mathrm{wf}}\right)\right\}} \\
t_{\mathrm{DA}} & =\frac{0.00633 k t}{\varphi \mu c_{t} A}
\end{aligned}
$$

\section{Normalized pressure integral typecurve analysis}

In case of NPI (Help Manual 2010), the normalized pressure replacing normalized rate was plotted against material-balance pseudo-time on a log-log scale of the same size as the typecurves, which are referred as the "data plot".

Normalized pressure, $\frac{\Delta p_{\mathrm{p}}}{q}=\frac{\left(p_{\mathrm{pi}}-p_{\mathrm{wf}}\right)}{q}$

Material balance pseudo time, $t_{\mathrm{ca}}$

$$
=\left\{\frac{\left(\mu_{\mathrm{g}} c_{\mathrm{g}}\right)_{j}}{q_{\mathrm{g}}}\right\} \int_{o}^{t}\left\{\frac{q_{\mathrm{g}}}{\mu_{\mathrm{gav}} c_{\mathrm{gav}}}\right\} \mathrm{d} t
$$

NPI typecurves were developed by dimensionless pressure $\left(p_{\mathrm{d}}\right)$ against dimensionless time $\left(t_{\mathrm{d}}\right)$ on a $\log -\log$ scale. 


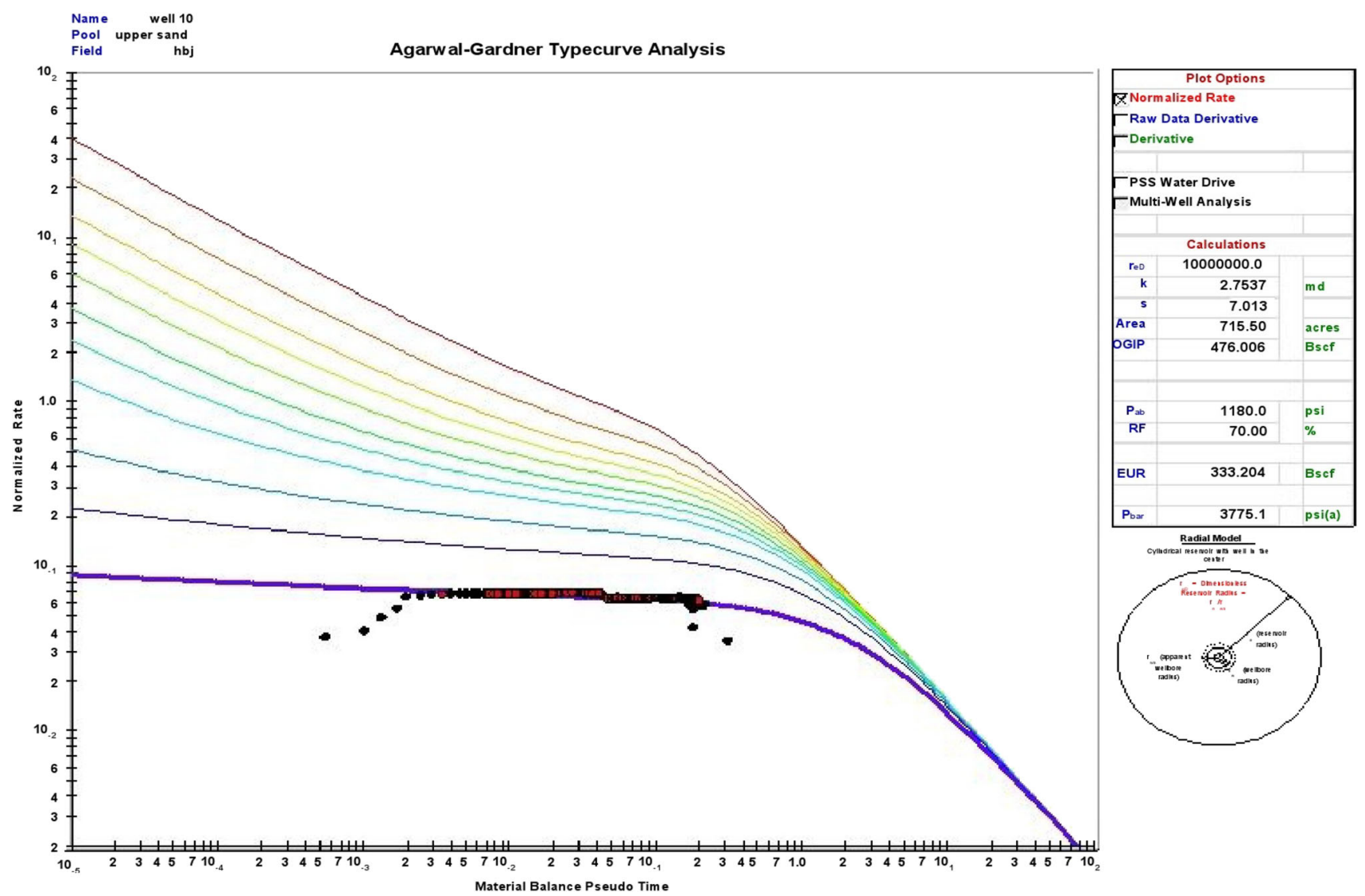

Fig. 7 Match between data plot for Well-10 and Agarwal-Gardner typecurve plot

$p_{\mathrm{D}}=\frac{1}{q_{\mathrm{D}}}=\frac{\left\{k h\left(p_{\mathrm{i}}-p_{\mathrm{wf}}\right)\right\}}{141.2 q B \mu}$

$t_{\mathrm{DA}}=\frac{0.00633 k t}{\varphi \mu c_{\mathrm{t}} A}$

\section{Analysis procedure}

After entering data in the software FEKETE, F.A.S.T.R$\mathrm{TA}^{\mathrm{TM}}$, instruction of the Help Manual was followed. According to the Help Manual, typecurve analysis is generally done by selecting a match point and reading its coordinates off the data plot $\left(\frac{q}{p}\right.$ and $\left.t_{\mathrm{ca}}\right)$ and off the typecurve plot ( $q_{\text {Dd }}$ and $\left.t_{\text {Dd }}\right)$ match. At the same time, the stem value $\frac{r_{\mathrm{e}}}{r_{\mathrm{w}}}$ of the matching curve is noted. In this study, data plot was moved over the typecurve plot to get the optimum match, while the axis of the two plots was kept parallel. Several positions of the several typecurves were tried and typecurve that fitted the data plot best was selected. From a curve match, the following reservoir parameters were found from the output of the software: GIIP, EUR, permeability and skin.

\section{Results and discussions}

Using three methods, graph of the match between data plot and individual typecurves are presented in Figs. 4, 5, 6 for Well-07 and Figs. 7, 8, 9 for Well-10, respectively. To get the optimum value of the results, three methods were averaged.

\section{GIIP and EUR}

GIIP of Well-07 is shown in Figs. 4, 5, 6 and of Well-10 is shown in Figs. 7, 8, 9, respectively. Table 1 shows the findings of GIIP values of Well-07 to be $435.082 \mathrm{Bcf}$ and of Well-10 is 475.242 Bcf, respectively, obtained from FEKETE, F.A.S.T.RTA ${ }^{\mathrm{TM}}$. It could be said that the difference of GIIP between these two wells is very small.

EUR of Well-07 is shown in Figs. 4, 5, 6 and of Well-10 is shown in Figs. 7, 8, 9, respectively. As shown in Table 2, the EUR value of Well-07 and Well-10 obtained from FEKETE, F.A.S.T.RTA ${ }^{\mathrm{TM}}$ was projected to be 

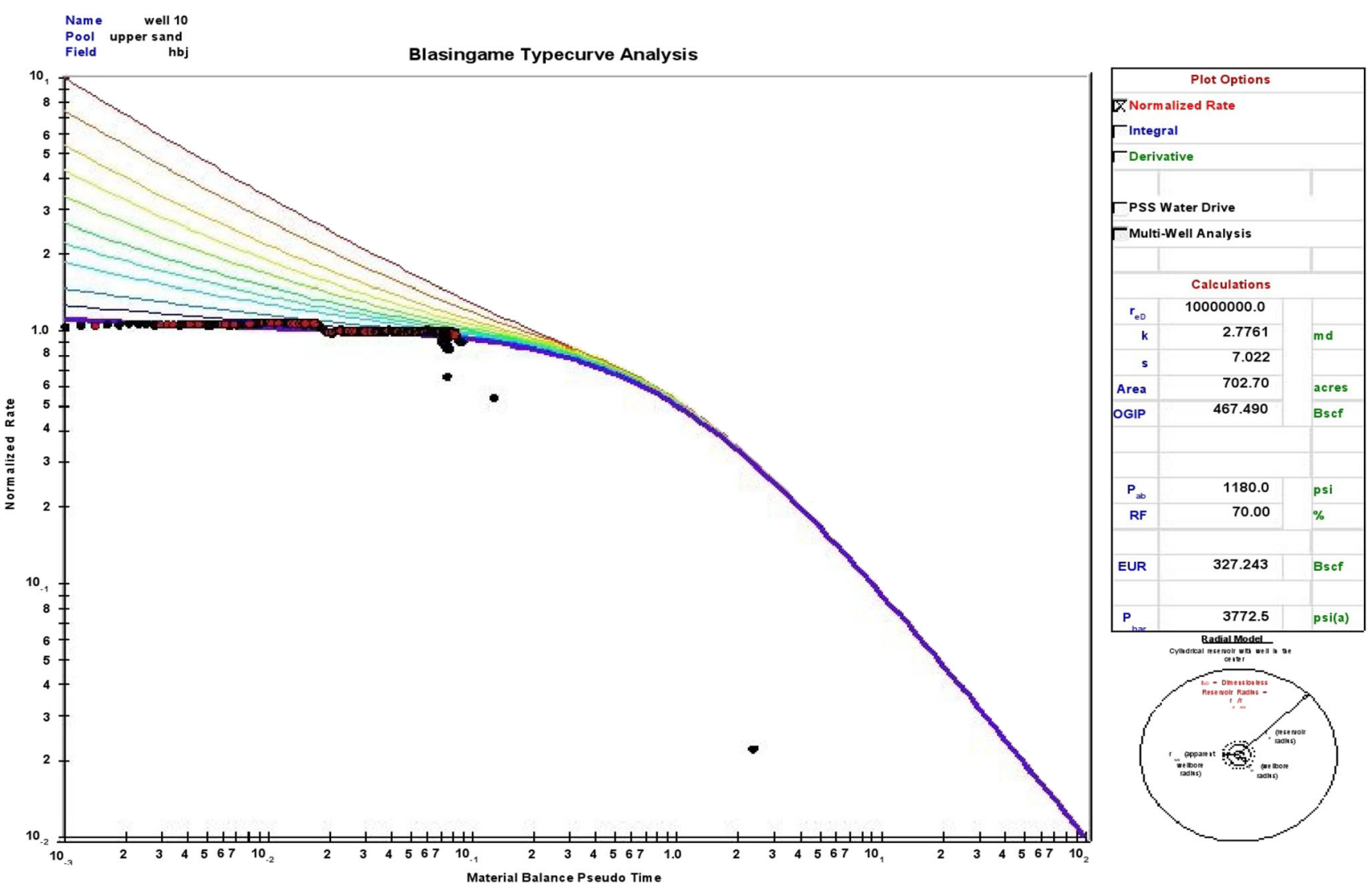

Fig. 8 Match between data plot for Well-10 and Blasingame typecurve plot

304.558 Bcf and 332.67 Bcf, respectively. This result suggests that only this amount of reserve from GIIP of these two wells mentioned above is commercially recoverable by primary recovery.

\section{Skin and permeability}

The value of permeability $(k)$ in the wellbore region of the Well-07 is illustrated in Figs. 4, 5, 6 and of Well-10 in Figs. 7, 8, 9, respectively. According to Table 3, permeability was estimated to 3.0396 md and 2.7839 md surrounding the Well-07 and Well-10, respectively, at the end of the year of 2007.

The value of the skin effect (dimensionless parameter) is depicted in Figs. 4, 5, 6 for Well-07 and for Well-10 in Figs. 7, 8, 9, respectively. As shown in Table 4, reservoir was damaged due to skin in surrounding region of the
Well-07 was valued by 7.017 and of Well-10 was 7.014, respectively.

\section{Comparison with previous studies}

Imam (2013) stated that the average permeability $(k)$ value of UGS of Habiganj gas field was found to be 2-4 darcy. But from this study, the permeability $(k)$ value of UGS of Habiganj gas field was obtained to $3.0396 \mathrm{md}$ and 2.7839 md surrounding Well-07 and Well-10, respectively. Additionally, the dimensionless value of skin factor (s) was estimated surrounding the Well-07 and Well-10 to 7.017 and 7.014, respectively. So, permeability near these two wellbore regions was substantially reduced as a result of the highly valued skin effect as recommended by Hawkins Jr. (1956). This reduced permeability causes an additional pressure drop across the altered zone. 


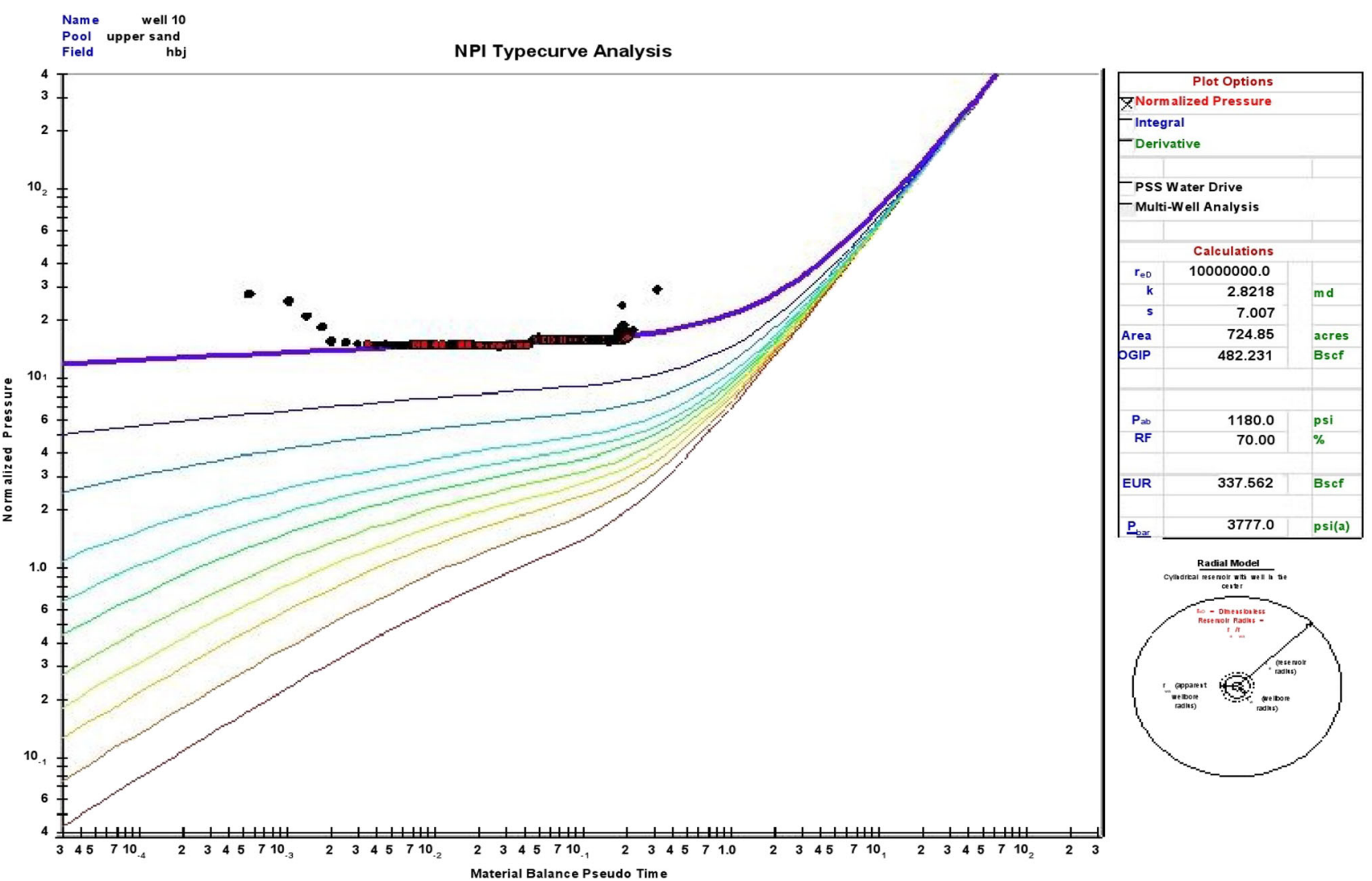

Fig. 9 Match between data plot for Well-10 and NPI typecurve plot

Table 1 GIIP of Well-07 and Well-10

\begin{tabular}{lllll}
\hline Well & Blasingame $(\mathrm{Bcf})$ & Agarwal-Gardner $(\mathrm{Bcf})$ & Normalized pressure integral $(\mathrm{Bcf})$ & Average $(\mathrm{Bcf})$ \\
\hline Well-07 & 432.944 & 434.186 & 438.117 & 435.082 \\
Well-10 & 467.49 & 476.006 & 482.231 & 475.242 \\
\hline
\end{tabular}

$B c f$ billion cubic feet

Table 2 EUR of Well-07 and Well-10

\begin{tabular}{lllll}
\hline Well & Blasingame $(\mathrm{Bcf})$ & Agarwal-Gardner $(\mathrm{Bcf})$ & Normalized pressure integral $(\mathrm{Bcf})$ & Average $(\mathrm{Bcf})$ \\
\hline Well-07 & 303.061 & 303.93 & 306.682 & 304.558 \\
Well-10 & 327.243 & 333.204 & 337.562 & 332.67 \\
\hline
\end{tabular}

$B c f$ billion cubic feet

Table 3 Permeability $(k)$ surrounding Well-07 and Well-10

\begin{tabular}{lllll}
\hline Well & Blasingame $(\mathrm{md})$ & Agarwal-Gardner $(\mathrm{md})$ & Normalized pressure integral $(\mathrm{md})$ & Average $(\mathrm{md})$ \\
\hline Well-07 & 3.0799 & 2.9880 & 3.0508 & 3.0396 \\
Well-10 & 2.7761 & 2.7537 & 2.8218 & 2.7839 \\
\hline
\end{tabular}

$m d$ millidarcy 
Table 4 Skin effect for Well-07 and Well-10

\begin{tabular}{lllll}
\hline Well & Blasingame & Agarwal-Gardner & Normalized pressure integral & Average \\
\hline Well-07 & 7.019 & 7.018 & 7.013 & 7.017 \\
Well-10 & 7.022 & 7.013 & 7.007 & 7.014 \\
\hline
\end{tabular}

Imam (2013)also mentioned that Petrobangla published a revised estimate of the Habiganj gas field based on RPS energy consultant showing estimated GIIP value of UGS is $3.68 \mathrm{Tcf}$ and EUR of $2.63 \mathrm{Tcf}$. This study found total estimated value of GIIP of these two wells to $910.324 \mathrm{Bcf}$ and EUR to 637.228 Bcf. So, it can be deduced that 637.228 Bcf of gas is recoverable by primary recovery from proven reserve of $2.63 \mathrm{Tcf}$ from the reservoir of Habiganj gas field, Bangladesh.

\section{Conclusions}

For the last few decades RTA, i.e., modern DCA, using typecurve is one of the developed and most used methods for analyzing petroleum reservoir. Averaging from three methods rate transient analyzing procedure, this study shows the estimated GIIP value of Well-07 and Well-10 of Habiganj gas field to $435.082 \mathrm{Bcf}$ and 475.242 Bcf, respectively, while the value of EUR of Well-07 and Well-10 was amounted to $304.558 \mathrm{Bcf}$ and 332.67 Bcf, respectively. The study shows the positive skin factor caused by reservoir damage for Well-07 and Well-10 was 7.017 and 7.014, reducing the value of formation permeability from 2-4 darcy to $3.0396 \mathrm{md}$ and 2.7839 md near wellbore region. This decreasing permeability due to formation damage retards the expected flow of fluids and, hence, the production.

Acknowledgments The authors would like to be thankful to Petrobangla for their support for providing the required data to conduct this research.

Open Access This article is distributed under the terms of the Creative Commons Attribution 4.0 International License (http:// creativecommons.org/licenses/by/4.0/), which permits unrestricted use, distribution, and reproduction in any medium, provided you give appropriate credit to the original author(s) and the source, provide a link to the Creative Commons license, and indicate if changes were made.
Table 5 Production data of Well-07 for the year of 2007

\begin{tabular}{|c|c|c|c|}
\hline Date $(\mathrm{M} / \mathrm{D} / \mathrm{Y})$ & Gas (MMScf) & FWHP (psig) & Water (Bbl) \\
\hline \multicolumn{4}{|l|}{ January 2007} \\
\hline $1 / 1 / 2007$ & 23.268 & 1600 & 4.101 \\
\hline $1 / 2 / 2007$ & 25.693 & 1555 & 4.296 \\
\hline $1 / 3 / 2007$ & 31.196 & 1545 & 4.516 \\
\hline $1 / 4 / 2007$ & 35.323 & 1542 & 4.164 \\
\hline $1 / 5 / 2007$ & 41.327 & 1525 & 4.755 \\
\hline $1 / 6 / 2007$ & 42.097 & 1486 & 4.875 \\
\hline $1 / 7 / 2007$ & 42.953 & 1468 & 4.894 \\
\hline $1 / 8 / 2007$ & 43.245 & 1437 & 5.573 \\
\hline $1 / 9 / 2007$ & 43.837 & 1412 & 5.548 \\
\hline $1 / 10 / 2007$ & 43.633 & 1414 & 5.12 \\
\hline $1 / 11 / 2007$ & 43.924 & 1411 & 5.183 \\
\hline $1 / 12 / 2007$ & 43.857 & 1411 & 5.164 \\
\hline $1 / 13 / 2007$ & 43.960 & 1410 & 5.259 \\
\hline $1 / 14 / 2007$ & 43.701 & 1414 & 5.101 \\
\hline $1 / 15 / 2007$ & 43.785 & 1413 & 4.995 \\
\hline $1 / 16 / 2007$ & 43.707 & 1414 & 8.423 \\
\hline $1 / 17 / 2007$ & 43.723 & 1414 & 7.831 \\
\hline $1 / 18 / 2007$ & 43.640 & 1415 & 7.561 \\
\hline $1 / 19 / 2007$ & 43.698 & 1414 & 7.819 \\
\hline $1 / 20 / 2007$ & 43.698 & 1414 & 7.876 \\
\hline $1 / 21 / 2007$ & 43.830 & 1413 & 7.787 \\
\hline $1 / 22 / 2007$ & 43.718 & 1414 & 7.781 \\
\hline $1 / 23 / 2007$ & 43.780 & 1413 & 7.63 \\
\hline $1 / 24 / 2007$ & 43.654 & 1414 & 7.813 \\
\hline $1 / 25 / 2007$ & 43.654 & 1414 & 7.787 \\
\hline $1 / 26 / 2007$ & 43.727 & 1414 & 5.133 \\
\hline $1 / 27 / 2007$ & 43.739 & 1414 & 9.064 \\
\hline $1 / 28 / 2007$ & 43.702 & 1414 & 8.247 \\
\hline $1 / 29 / 2007$ & 43.721 & 1414 & 7.769 \\
\hline $1 / 30 / 2007$ & 43.721 & 1414 & 5.026 \\
\hline $1 / 31 / 2007$ & 43.698 & 1414 & 5.089 \\
\hline \multicolumn{4}{|l|}{ February 2007} \\
\hline $2 / 1 / 2007$ & 43.731 & 1414 & 5.775 \\
\hline $2 / 2 / 2007$ & 43.644 & 1415 & 5.837 \\
\hline
\end{tabular}

\section{Appendix 1}

See Table 5 and Figs. 10, 11, 12. 
Table 5 continued

\begin{tabular}{|c|c|c|c|c|c|c|c|}
\hline Date $(\mathrm{M} / \mathrm{D} / \mathrm{Y})$ & Gas (MMScf) & FWHP (psig) & Water (Bbl) & Date $(\mathrm{M} / \mathrm{D} / \mathrm{Y})$ & Gas (MMScf) & FWHP (psig) & Water (Bbl) \\
\hline $2 / 3 / 2007$ & 43.682 & 1414 & 7.586 & $3 / 22 / 2007$ & 40.257 & 1445 & 7.926 \\
\hline $2 / 4 / 2007$ & 43.931 & 1412 & 6.882 & $3 / 23 / 2007$ & 41.057 & 1443 & 7.756 \\
\hline $2 / 5 / 2007$ & 43.917 & 1412 & 6.737 & $3 / 24 / 2007$ & 40.505 & 1450 & 5.309 \\
\hline $2 / 6 / 2007$ & 43.878 & 1412 & 5.925 & $3 / 25 / 2007$ & 40.136 & 1455 & 4.642 \\
\hline $2 / 7 / 2007$ & 43.939 & 1412 & 6.051 & $3 / 26 / 2007$ & 39.723 & 1458 & 4.711 \\
\hline $2 / 8 / 2007$ & 43.967 & 1411 & 5.429 & $3 / 27 / 2007$ & 40.31 & 1452 & 5.076 \\
\hline $2 / 9 / 2007$ & 43.764 & 1413 & 5.548 & $3 / 28 / 2007$ & 40.392 & 1451 & 7.97 \\
\hline $2 / 10 / 2007$ & 43.710 & 1414 & 4.894 & $3 / 29 / 2007$ & 40.312 & 1452 & 4.56 \\
\hline $2 / 11 / 2007$ & 43.808 & 1413 & 5.02 & $3 / 30 / 2007$ & 40.371 & 1451 & 4.636 \\
\hline $2 / 12 / 2007$ & 43.732 & 1414 & 5.429 & $3 / 31 / 2007$ & 40.436 & 1451 & 6.064 \\
\hline $2 / 13 / 2007$ & 43.795 & 1413 & 6.02 & April 2007 & & & \\
\hline $2 / 14 / 2007$ & 43.818 & 1413 & 5.894 & $4 / 1 / 2007$ & 40.241 & 1459 & 6.429 \\
\hline $2 / 15 / 2007$ & 43.793 & 1413 & 5.101 & $4 / 2 / 2007$ & 40.466 & 1456 & 6.429 \\
\hline $2 / 16 / 2007$ & 43.798 & 1413 & 4.938 & $4 / 3 / 2007$ & 40.281 & 1458 & 6.429 \\
\hline $2 / 17 / 2007$ & 43.792 & 1413 & 5.391 & $4 / 4 / 2007$ & 40.338 & 1458 & 6.429 \\
\hline $2 / 18 / 2007$ & 43.786 & 1413 & 4.888 & $4 / 5 / 2007$ & 40.332 & 1458 & 6.429 \\
\hline $2 / 19 / 2007$ & 43.753 & 1413 & 6.611 & $4 / 6 / 2007$ & 40.298 & 1458 & 6.429 \\
\hline $2 / 20 / 2007$ & 43.896 & 1412 & 5.221 & $4 / 7 / 2007$ & 39.81 & 1463 & 6.429 \\
\hline $2 / 21 / 2007$ & 43.779 & 1413 & 5.385 & $4 / 8 / 2007$ & 40.698 & 1455 & 6.429 \\
\hline $2 / 22 / 2007$ & 43.807 & 1413 & 5.265 & 4/9/2007 & 39.626 & 1465 & 6.429 \\
\hline $2 / 23 / 2007$ & 43.811 & 1413 & 8.159 & $4 / 10 / 2007$ & 39.961 & 1461 & 6.429 \\
\hline $2 / 24 / 2007$ & 43.713 & 1414 & 6.58 & $4 / 11 / 2007$ & 40.403 & 1457 & 6.429 \\
\hline $2 / 25 / 2007$ & 43.715 & 1414 & 6.278 & $4 / 12 / 2007$ & 40.4 & 1457 & 6.429 \\
\hline $2 / 26 / 2007$ & 43.807 & 1413 & 6.12 & $4 / 13 / 2007$ & 40.162 & 1459 & 6.429 \\
\hline $2 / 27 / 2007$ & 43.794 & 1413 & 6.196 & $4 / 14 / 2007$ & 40.096 & 1460 & 6.429 \\
\hline $2 / 28 / 2007$ & 43.756 & 1413 & 5.309 & $4 / 15 / 2007$ & 40.275 & 1458 & 6.429 \\
\hline March 2007 & & & & $4 / 16 / 2007$ & 39.972 & 1461 & 6.429 \\
\hline $3 / 1 / 2007$ & 43.744 & 1416 & 6.014 & $4 / 17 / 2007$ & 40.274 & 1458 & 6.429 \\
\hline $3 / 2 / 2007$ & 43.34 & 1418 & 5.315 & $4 / 18 / 2007$ & 39.678 & 1462 & 6.429 \\
\hline $3 / 3 / 2007$ & 43.754 & 1420 & 6.095 & $4 / 19 / 2007$ & 39.717 & 1462 & 6.429 \\
\hline $3 / 4 / 2007$ & 43.728 & 1421 & 5.718 & $4 / 20 / 2007$ & 39.796 & 1461 & 6.429 \\
\hline $3 / 5 / 2007$ & 43.836 & 1420 & 5.8 & $4 / 21 / 2007$ & 40.069 & 1460 & 6.429 \\
\hline $3 / 6 / 2007$ & 43.758 & 1420 & 5.674 & $4 / 22 / 2007$ & 39.924 & 1462 & 6.429 \\
\hline $3 / 7 / 2007$ & 43.751 & 1420 & 5.73 & $4 / 23 / 2007$ & 39.708 & 1464 & 6.429 \\
\hline $3 / 8 / 2007$ & 43.814 & 1420 & 5.611 & $4 / 24 / 2007$ & 40.151 & 1459 & 6.429 \\
\hline 3/9/2007 & 42.935 & 1425 & 5.498 & $4 / 25 / 2007$ & 40.176 & 1459 & 6.429 \\
\hline $3 / 10 / 2007$ & 43.824 & 1420 & 5.227 & $4 / 26 / 2007$ & 40.205 & 1459 & 6.429 \\
\hline $3 / 11 / 2007$ & 43.788 & 1420 & 5.101 & $4 / 27 / 2007$ & 39.736 & 1463 & 6.429 \\
\hline $3 / 12 / 2007$ & 43.863 & 1419 & 5.819 & $4 / 28 / 2007$ & 40.228 & 1459 & 6.429 \\
\hline $3 / 13 / 2007$ & 43.838 & 1420 & 5.624 & $4 / 29 / 2007$ & 40.272 & 1458 & 6.429 \\
\hline $3 / 14 / 2007$ & 43.882 & 1419 & 5.762 & $4 / 30 / 2007$ & 39.846 & 1463 & 6.429 \\
\hline $3 / 15 / 2007$ & 43.871 & 1419 & 5.397 & May 2007 & & & \\
\hline $3 / 16 / 2007$ & 43.261 & 1422 & 3.535 & $5 / 1 / 2007$ & 40.056 & 1459 & 4.06334 \\
\hline $3 / 17 / 2007$ & 43.365 & 1421 & 6.479 & $5 / 2 / 2007$ & 40.267 & 1457 & 3.99415 \\
\hline $3 / 18 / 2007$ & 43.655 & 1421 & 5.63 & $5 / 3 / 2007$ & 40.401 & 1456 & 4.4659 \\
\hline $3 / 19 / 2007$ & 43.915 & 1420 & 5.598 & $5 / 4 / 2007$ & 40.139 & 1459 & 4.10108 \\
\hline $3 / 20 / 2007$ & 42.358 & 1420 & 7.775 & $5 / 5 / 2007$ & 40.158 & 1458 & 4.32123 \\
\hline $3 / 21 / 2007$ & 41.017 & 1436 & 5.68 & $5 / 6 / 2007$ & 40.334 & 1457 & 4.44074 \\
\hline
\end{tabular}

Table 5 continued 
Table 5 continued

\begin{tabular}{|c|c|c|c|}
\hline Date $(\mathrm{M} / \mathrm{D} / \mathrm{Y})$ & Gas (MMScf) & FWHP (psig) & Water $(\mathrm{Bbl})$ \\
\hline $5 / 7 / 2007$ & 40.066 & 1459 & 4.11995 \\
\hline $5 / 8 / 2007$ & 40.271 & 1457 & 4.20172 \\
\hline $5 / 9 / 2007$ & 40.215 & 1458 & 4.36526 \\
\hline $5 / 10 / 2007$ & 40.053 & 1459 & 4.28349 \\
\hline $5 / 11 / 2007$ & 40.056 & 1459 & 4.30865 \\
\hline $5 / 12 / 2007$ & 40.113 & 1459 & 4.39671 \\
\hline $5 / 13 / 2007$ & 40.286 & 1457 & 4.20172 \\
\hline $5 / 14 / 2007$ & 40.310 & 1457 & 4.14511 \\
\hline $5 / 15 / 2007$ & 40.281 & 1457 & 4.27091 \\
\hline $5 / 16 / 2007$ & 40.293 & 1457 & 4.2143 \\
\hline $5 / 17 / 2007$ & 40.185 & 1458 & 4.09479 \\
\hline $5 / 18 / 2007$ & 40.219 & 1458 & 4.37784 \\
\hline $5 / 19 / 2007$ & 40.376 & 1456 & 3.95012 \\
\hline $5 / 20 / 2007$ & 40.278 & 1457 & 4.28349 \\
\hline $5 / 21 / 2007$ & 40.042 & 1460 & 4.23317 \\
\hline $5 / 22 / 2007$ & 40.282 & 1457 & 4.35897 \\
\hline $5 / 23 / 2007$ & 40.041 & 1460 & 4.24575 \\
\hline $5 / 24 / 2007$ & 40.331 & 1457 & 4.25833 \\
\hline $5 / 25 / 2007$ & 40.246 & 1458 & 4.09479 \\
\hline $5 / 26 / 2007$ & 40.240 & 1458 & 4.31494 \\
\hline $5 / 27 / 2007$ & 40.105 & 1459 & 4.30865 \\
\hline $5 / 28 / 2007$ & 40.231 & 1458 & 4.28978 \\
\hline $5 / 29 / 2007$ & 40.084 & 1459 & 4.17027 \\
\hline $5 / 30 / 2007$ & 39.996 & 1460 & 4.32752 \\
\hline $5 / 31 / 2007$ & 40.534 & 1459 & 3.35886 \\
\hline \multicolumn{4}{|l|}{ June 2007} \\
\hline $6 / 1 / 2007$ & 40.166 & 1450 & 4.47219 \\
\hline $6 / 2 / 2007$ & 40.204 & 1440 & 4.61686 \\
\hline $6 / 3 / 2007$ & 40.095 & 1437 & 4.57912 \\
\hline $6 / 4 / 2007$ & 40.251 & 1436 & 4.79298 \\
\hline $6 / 5 / 2007$ & 40.278 & 1435 & 4.57912 \\
\hline $6 / 6 / 2007$ & 40.121 & 1437 & 4.5288 \\
\hline $6 / 7 / 2007$ & 40.128 & 1437 & 4.52251 \\
\hline $6 / 8 / 2007$ & 40.387 & 1434 & 4.70492 \\
\hline $6 / 9 / 2007$ & 40.293 & 1435 & 4.74895 \\
\hline $6 / 10 / 2007$ & 40.248 & 1436 & 4.63573 \\
\hline $6 / 11 / 2007$ & 40.331 & 1435 & 4.79298 \\
\hline $6 / 12 / 2007$ & 40.013 & 1438 & 4.52251 \\
\hline $6 / 13 / 2007$ & 40.244 & 1436 & 4.49106 \\
\hline $6 / 14 / 2007$ & 40.246 & 1436 & 4.56025 \\
\hline $6 / 15 / 2007$ & 40.229 & 1436 & 4.66718 \\
\hline $6 / 16 / 2007$ & 40.383 & 1434 & 4.79298 \\
\hline $6 / 17 / 2007$ & 40.392 & 1436 & 3.95641 \\
\hline $6 / 18 / 2007$ & 40.322 & 1435 & 3.94383 \\
\hline $6 / 19 / 2007$ & 40.478 & 1433 & 4.55396 \\
\hline $6 / 20 / 2007$ & 40.640 & 1432 & 4.75524 \\
\hline $6 / 21 / 2007$ & 40.455 & 1433 & 3.96899 \\
\hline $6 / 22 / 2007$ & 40.341 & 1435 & 4.50993 \\
\hline
\end{tabular}

Table 5 continued

\begin{tabular}{|c|c|c|c|}
\hline Date $(\mathrm{M} / \mathrm{D} / \mathrm{Y})$ & Gas (MMScf) & FWHP (psig) & Water (Bbl) \\
\hline $6 / 23 / 2007$ & 40.298 & 1435 & 4.38413 \\
\hline $6 / 24 / 2007$ & 40.370 & 1434 & 4.30865 \\
\hline $6 / 25 / 2007$ & 40.513 & 1433 & 4.52251 \\
\hline $6 / 26 / 2007$ & 40.462 & 1433 & 4.31494 \\
\hline $6 / 27 / 2007$ & 40.414 & 1434 & 4.80556 \\
\hline $6 / 28 / 2007$ & 40.093 & 1437 & 4.77411 \\
\hline $6 / 29 / 2007$ & 40.324 & 1435 & 4.79298 \\
\hline $6 / 30 / 2007$ & 40.190 & 1436 & 4.74266 \\
\hline \multicolumn{4}{|l|}{ July 2007} \\
\hline $7 / 1 / 2007$ & 40.154 & 1430 & 4.64831 \\
\hline $7 / 2 / 2007$ & 40.329 & 1425 & 4.55396 \\
\hline $7 / 3 / 2007$ & 40.337 & 1422 & 4.62315 \\
\hline $7 / 4 / 2007$ & 40.375 & 1420 & 4.54138 \\
\hline $7 / 5 / 2007$ & 40.353 & 1417 & 4.58541 \\
\hline $7 / 6 / 2007$ & 40.295 & 1415 & 4.63573 \\
\hline $7 / 7 / 2007$ & 40.342 & 1414 & 4.64831 \\
\hline $7 / 8 / 2007$ & 40.353 & 1413 & 4.81814 \\
\hline $7 / 9 / 2007$ & 40.324 & 1414 & 4.62944 \\
\hline $7 / 10 / 2007$ & 40.463 & 1412 & 4.64202 \\
\hline $7 / 11 / 2007$ & 40.458 & 1412 & 4.61686 \\
\hline $7 / 12 / 2007$ & 40.566 & 1411 & 4.62315 \\
\hline $7 / 13 / 2007$ & 40.412 & 1413 & 4.22688 \\
\hline $7 / 14 / 2007$ & 40.543 & 1412 & 4.52251 \\
\hline $7 / 15 / 2007$ & 40.453 & 1412 & 4.74266 \\
\hline $7 / 16 / 2007$ & 40.132 & 1416 & 4.74266 \\
\hline $7 / 17 / 2007$ & 40.419 & 1413 & 4.70492 \\
\hline $7 / 18 / 2007$ & 40.087 & 1416 & 4.73008 \\
\hline $7 / 19 / 2007$ & 40.117 & 1416 & 4.67976 \\
\hline $7 / 20 / 2007$ & 40.105 & 1416 & 4.64831 \\
\hline $7 / 21 / 2007$ & 40.164 & 1415 & 4.48477 \\
\hline $7 / 22 / 2007$ & 40.334 & 1414 & 4.51622 \\
\hline $7 / 23 / 2007$ & 39.840 & 1419 & 4.57283 \\
\hline $7 / 24 / 2007$ & 39.921 & 1418 & 4.56025 \\
\hline $7 / 25 / 2007$ & 39.694 & 1420 & 4.06334 \\
\hline $7 / 26 / 2007$ & 40.291 & 1414 & 4.76153 \\
\hline $7 / 27 / 2007$ & 40.130 & 1416 & 4.71121 \\
\hline $7 / 28 / 2007$ & 40.035 & 1417 & 4.49735 \\
\hline $7 / 29 / 2007$ & 39.953 & 1417 & 4.61057 \\
\hline $7 / 30 / 2007$ & 40.261 & 1414 & 4.49106 \\
\hline $7 / 31 / 2007$ & 40.239 & 1415 & 4.59799 \\
\hline \multicolumn{4}{|l|}{ August 2007} \\
\hline $8 / 1 / 2007$ & 39.945 & 1430 & 4.37784 \\
\hline $8 / 2 / 2007$ & 40.235 & 1438 & 4.49106 \\
\hline $8 / 3 / 2007$ & 38.000 & 1456 & 4.1514 \\
\hline $8 / 4 / 2007$ & 40.151 & 1445 & 4.67347 \\
\hline $8 / 5 / 2007$ & 40.000 & 1445 & 4.61686 \\
\hline $8 / 6 / 2007$ & 39.846 & 1445 & 4.76153 \\
\hline $8 / 7 / 2007$ & 40.038 & 1456 & 4.56654 \\
\hline
\end{tabular}


Table 5 continued

\begin{tabular}{|c|c|c|c|c|c|c|c|}
\hline Date $(\mathrm{M} / \mathrm{D} / \mathrm{Y})$ & Gas (MMScf) & FWHP (psig) & Water (Bbl) & Date $(\mathrm{M} / \mathrm{D} / \mathrm{Y})$ & Gas (MMScf) & FWHP (psig) & Water (Bbl) \\
\hline $8 / 8 / 2007$ & 39.955 & 1456 & 4.39671 & $9 / 24 / 2007$ & 40.502 & 1407 & 4.93136 \\
\hline $8 / 9 / 2007$ & 39.875 & 1457 & 4.69863 & $9 / 25 / 2007$ & 40.130 & 1411 & 4.70492 \\
\hline $8 / 10 / 2007$ & 40.094 & 1455 & 4.1514 & $9 / 26 / 2007$ & 40.366 & 1408 & 4.69234 \\
\hline $8 / 11 / 2007$ & 40.190 & 1456 & 4.28978 & $9 / 27 / 2007$ & 40.229 & 1409 & 4.79298 \\
\hline $8 / 12 / 2007$ & 40.023 & 1456 & 4.61057 & $9 / 28 / 2007$ & 40.388 & 1408 & 4.88104 \\
\hline $8 / 13 / 2007$ & 40.225 & 1454 & 4.7175 & $9 / 29 / 2007$ & 40.133 & 1411 & 4.13253 \\
\hline $8 / 14 / 2007$ & 39.832 & 1458 & 4.44074 & $9 / 30 / 2007$ & 40.368 & 1408 & 4.43445 \\
\hline $8 / 15 / 2007$ & 40.044 & 1456 & 4.52251 & October 2007 & & & \\
\hline $8 / 16 / 2007$ & 40.109 & 1455 & 4.64202 & $10 / 1 / 2007$ & 40.855 & 1406 & 4.01931 \\
\hline $8 / 17 / 2007$ & 39.958 & 1456 & 4.74266 & $10 / 2 / 2007$ & 40.438 & 1408 & 4.70492 \\
\hline $8 / 18 / 2007$ & 40.010 & 1456 & 4.87475 & $10 / 3 / 2007$ & 40.227 & 1410 & 4.79927 \\
\hline $8 / 19 / 2007$ & 40.190 & 1454 & 4.70492 & $10 / 4 / 2007$ & 40.439 & 1408 & 4.56654 \\
\hline $8 / 20 / 2007$ & 40.296 & 1453 & 4.60428 & $10 / 5 / 2007$ & 40.102 & 1410 & 4.89991 \\
\hline $8 / 21 / 2007$ & 40.423 & 1452 & 4.73008 & $10 / 6 / 2007$ & 40.066 & 1411 & 4.98797 \\
\hline $8 / 22 / 2007$ & 40.042 & 1456 & 4.56654 & $10 / 7 / 2007$ & 40.563 & 1406 & 4.48477 \\
\hline $8 / 23 / 2007$ & 39.885 & 1457 & 4.42187 & $10 / 8 / 2007$ & 40.343 & 1409 & 4.66718 \\
\hline $8 / 24 / 2007$ & 40.083 & 1455 & 4.63573 & $10 / 9 / 2007$ & 40.459 & 1407 & 4.32752 \\
\hline $8 / 25 / 2007$ & 40.079 & 1455 & 4.64831 & $10 / 10 / 2007$ & 39.981 & 1412 & 4.56025 \\
\hline $8 / 26 / 2007$ & 40.206 & 1454 & 4.67976 & $10 / 11 / 2007$ & 39.914 & 1414 & 3.98786 \\
\hline $8 / 27 / 2007$ & 39.977 & 1456 & 4.50364 & $10 / 12 / 2007$ & 39.825 & 1415 & 4.39671 \\
\hline $8 / 28 / 2007$ & 39.958 & 1456 & 4.67976 & $10 / 13 / 2007$ & 36.469 & 1440 & 4.22059 \\
\hline $8 / 29 / 2007$ & 40.421 & 1452 & 4.05705 & $10 / 14 / 2007$ & 36.504 & 1440 & 4.13882 \\
\hline $8 / 30 / 2007$ & 40.324 & 1453 & 4.64831 & $10 / 15 / 2007$ & 33.905 & 1455 & 3.95641 \\
\hline $8 / 31 / 2007$ & 40.539 & 1451 & 4.63573 & $10 / 16 / 2007$ & 34.815 & 1450 & 4.11366 \\
\hline September 2007 & & & & $10 / 17 / 2007$ & 39.416 & 1415 & 4.50364 \\
\hline $9 / 1 / 2007$ & 40.262 & 1435 & 4.60428 & $10 / 18 / 2007$ & 40.171 & 1411 & 4.23317 \\
\hline $9 / 2 / 2007$ & 40.159 & 1425 & 4.64831 & $10 / 19 / 2007$ & 39.917 & 1413 & 4.63573 \\
\hline $9 / 3 / 2007$ & 40.267 & 1420 & 4.81814 & $10 / 20 / 2007$ & 39.974 & 1412 & 4.68605 \\
\hline $9 / 4 / 2007$ & 39.895 & 1418 & 4.73008 & $10 / 21 / 2007$ & 40.001 & 1412 & 4.74266 \\
\hline $9 / 5 / 2007$ & 40.457 & 1413 & 4.79298 & $10 / 22 / 2007$ & 40.126 & 1411 & 4.07592 \\
\hline $9 / 6 / 2007$ & 40.171 & 1411 & 4.71121 & $10 / 23 / 2007$ & 39.999 & 1412 & 4.72379 \\
\hline $9 / 7 / 2007$ & 40.040 & 1412 & 4.7804 & $10 / 24 / 2007$ & 39.940 & 1413 & 4.63573 \\
\hline $9 / 8 / 2007$ & 40.405 & 1410 & 5.54778 & $10 / 25 / 2007$ & 39.822 & 1414 & 4.4659 \\
\hline 9/9/2007 & 40.270 & 1409 & 4.81814 & $10 / 26 / 2007$ & 40.027 & 1412 & 4.41558 \\
\hline $9 / 10 / 2007$ & 40.221 & 1410 & 4.49735 & $10 / 27 / 2007$ & 40.213 & 1410 & 4.69234 \\
\hline $9 / 11 / 2007$ & 40.331 & 1409 & 4.75524 & $10 / 28 / 2007$ & 40.060 & 1411 & 4.76782 \\
\hline $9 / 12 / 2007$ & 40.509 & 1407 & 4.69234 & $10 / 29 / 2007$ & 40.081 & 1411 & 4.58541 \\
\hline $9 / 13 / 2007$ & 40.469 & 1407 & 4.66718 & $10 / 30 / 2007$ & 39.939 & 1413 & 4.76153 \\
\hline $9 / 14 / 2007$ & 40.483 & 1407 & 4.64831 & $10 / 31 / 2007$ & 40.399 & 1408 & 4.81814 \\
\hline $9 / 15 / 2007$ & 40.425 & 1408 & 4.66089 & November 2007 & & & \\
\hline $9 / 16 / 2007$ & 40.243 & 1410 & 4.49106 & $11 / 1 / 2007$ & 40.160 & 1425 & 4.64831 \\
\hline $9 / 17 / 2007$ & 40.512 & 1408 & 9.47903 & $11 / 2 / 2007$ & 40.205 & 1440 & 4.47219 \\
\hline $9 / 18 / 2007$ & 40.538 & 1407 & 8.96325 & $11 / 3 / 2007$ & 40.093 & 1454 & 4.70492 \\
\hline $9 / 19 / 2007$ & 40.081 & 1409 & 7.51655 & $11 / 4 / 2007$ & 39.964 & 1460 & 4.50364 \\
\hline $9 / 20 / 2007$ & 40.062 & 1409 & 4.83701 & $11 / 5 / 2007$ & 40.127 & 1464 & 4.45961 \\
\hline $9 / 21 / 2007$ & 40.566 & 1406 & 5.68616 & $11 / 6 / 2007$ & 39.986 & 1470 & 4.56654 \\
\hline $9 / 22 / 2007$ & 40.180 & 1410 & 3.82432 & $11 / 7 / 2007$ & 40.022 & 1475 & 4.69234 \\
\hline $9 / 23 / 2007$ & 40.052 & 1411 & 4.64831 & $11 / 8 / 2007$ & 39.947 & 1480 & 4.44074 \\
\hline
\end{tabular}

Table 5 continued 
Table 5 continued

\begin{tabular}{|c|c|c|c|}
\hline Date $(\mathrm{M} / \mathrm{D} / \mathrm{Y})$ & Gas (MMScf) & FWHP (psig) & Water (Bbl) \\
\hline $11 / 9 / 2007$ & 40.050 & 1486 & 4.61057 \\
\hline $11 / 10 / 2007$ & 40.138 & 1485 & 4.41558 \\
\hline $11 / 11 / 2007$ & 40.074 & 1485 & 4.42187 \\
\hline $11 / 12 / 2007$ & 39.945 & 1487 & 4.4659 \\
\hline $11 / 13 / 2007$ & 39.865 & 1487 & 4.45961 \\
\hline $11 / 14 / 2007$ & 40.139 & 1485 & 4.64831 \\
\hline $11 / 15 / 2007$ & 37.663 & 1493 & 2.78018 \\
\hline $11 / 16 / 2007$ & 38.099 & 1492 & 4.89991 \\
\hline $11 / 17 / 2007$ & 39.769 & 1488 & 4.42816 \\
\hline $11 / 18 / 2007$ & 40.361 & 1482 & 4.55396 \\
\hline $11 / 19 / 2007$ & 40.252 & 1483 & 4.67347 \\
\hline $11 / 20 / 2007$ & 40.187 & 1484 & 4.86217 \\
\hline $11 / 21 / 2007$ & 40.290 & 1483 & 4.61686 \\
\hline $11 / 22 / 2007$ & 40.198 & 1484 & 4.57912 \\
\hline $11 / 23 / 2007$ & 40.169 & 1484 & 4.44703 \\
\hline $11 / 24 / 2007$ & 40.422 & 1482 & 4.67976 \\
\hline $11 / 25 / 2007$ & 40.189 & 1484 & 4.57912 \\
\hline $11 / 26 / 2007$ & 40.156 & 1484 & 4.59799 \\
\hline $11 / 27 / 2007$ & 40.069 & 1485 & 4.72379 \\
\hline $11 / 28 / 2007$ & 40.234 & 1484 & 4.7804 \\
\hline $11 / 29 / 2007$ & 40.194 & 1484 & 4.70492 \\
\hline $11 / 30 / 2007$ & 40.264 & 1483 & 4.57283 \\
\hline \multicolumn{4}{|l|}{ December 2007} \\
\hline $12 / 1 / 2007$ & 40.441 & 1482 & 4.57912 \\
\hline $12 / 2 / 2007$ & 40.316 & 1470 & 4.73637 \\
\hline $12 / 3 / 2007$ & 40.398 & 1465 & 4.18285 \\
\hline $12 / 4 / 2007$ & 40.380 & 1460 & 4.29607 \\
\hline $12 / 5 / 2007$ & 39.976 & 1455 & 4.42187 \\
\hline $12 / 6 / 2007$ & 39.892 & 1450 & 4.66718 \\
\hline $12 / 7 / 2007$ & 40.004 & 1446 & 4.61057 \\
\hline $12 / 8 / 2007$ & 39.379 & 1452 & 4.47848 \\
\hline $12 / 9 / 2007$ & 39.807 & 1448 & 4.61686 \\
\hline $12 / 10 / 2007$ & 39.580 & 1450 & 4.52251 \\
\hline $12 / 11 / 2007$ & 39.783 & 1448 & 4.58541 \\
\hline $12 / 12 / 2007$ & 40.164 & 1444 & 4.62944 \\
\hline $12 / 13 / 2007$ & 39.997 & 1446 & 4.41558 \\
\hline $12 / 14 / 2007$ & 40.424 & 1442 & 4.79298 \\
\hline $12 / 15 / 2007$ & 40.232 & 1444 & 4.54138 \\
\hline $12 / 16 / 2007$ & 40.015 & 1446 & 4.42187 \\
\hline $12 / 17 / 2007$ & 40.372 & 1442 & 4.45332 \\
\hline $12 / 18 / 2007$ & 40.364 & 1442 & 4.53509 \\
\hline $12 / 19 / 2007$ & 40.342 & 1443 & 4.50993 \\
\hline $12 / 20 / 2007$ & 36.723 & 1485 & 3.60417 \\
\hline $12 / 21 / 2007$ & 38.039 & 1479 & 3.75513 \\
\hline $12 / 22 / 2007$ & 38.698 & 1477 & 4.13253 \\
\hline $12 / 23 / 2007$ & 38.891 & 1470 & 4.44703 \\
\hline $12 / 24 / 2007$ & 39.896 & 1462 & 4.2143 \\
\hline $12 / 25 / 2007$ & 39.896 & 1449 & 5.84341 \\
\hline
\end{tabular}

Table 5 continued

\begin{tabular}{llll}
\hline Date $(\mathrm{M} / \mathrm{D} / \mathrm{Y})$ & Gas $(\mathrm{MMScf})$ & FWHP $(\mathrm{psig})$ & Water $(\mathrm{Bbl})$ \\
\hline $12 / 26 / 2007$ & 39.164 & 1455 & 4.403 \\
$12 / 27 / 2007$ & 40.044 & 1446 & 4.83072 \\
$12 / 28 / 2007$ & 39.872 & 1447 & 4.4659 \\
$12 / 29 / 2007$ & 39.675 & 1449 & 4.61057 \\
$12 / 30 / 2007$ & 36.418 & 1488 & 4.24575 \\
$12 / 31 / 2007$ & 40.067 & 1445 & 4.66089 \\
\hline
\end{tabular}

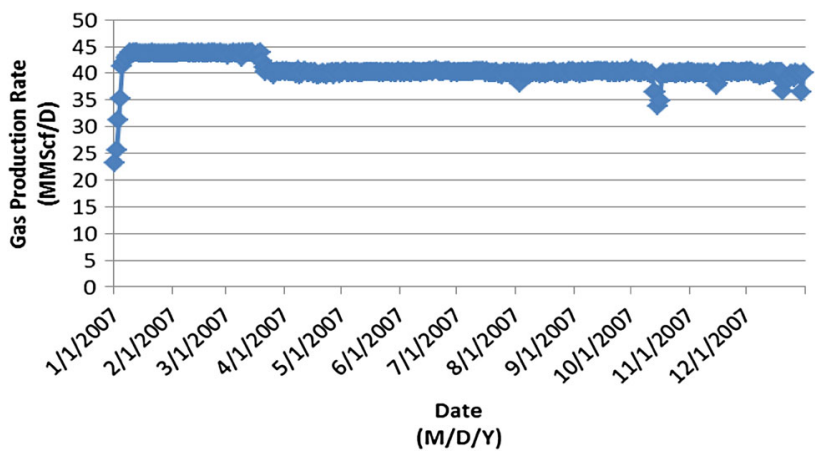

Fig. 10 Graphical presentation of gas production rate of Well-07 during the year of 2007

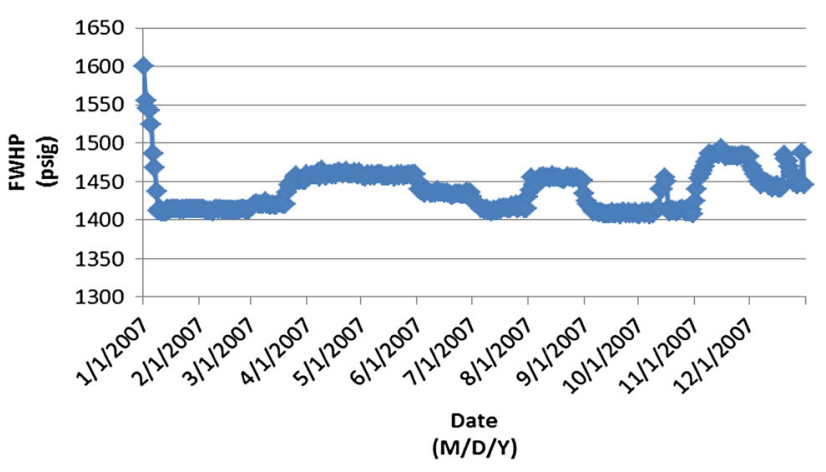

Fig. 11 Graphical presentation of flowing wellhead pressure (FWHP) of Well-07 during the year of 2007

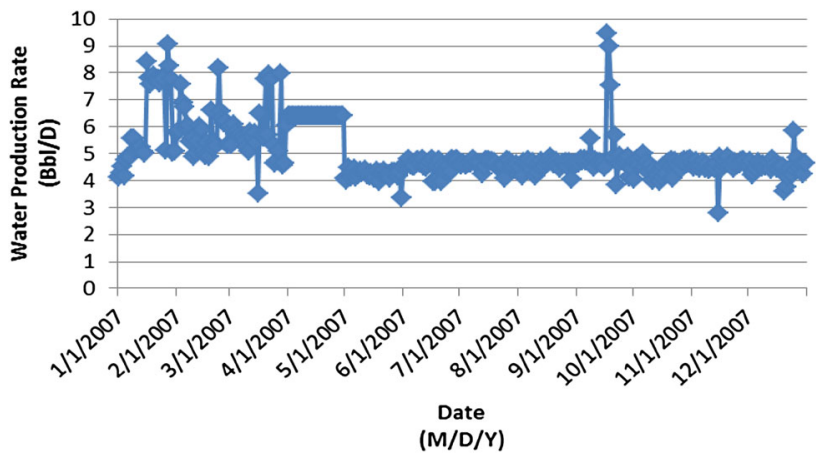

Fig. 12 Graphical presentation of water production rate of Well-07 during the year of 2007 


\section{Appendix 2}

See Tables 6 and Figs. 13, 14, 15.

Table 6 Production data of Well-10 for the year of 2007

\begin{tabular}{|c|c|c|c|}
\hline Date $(\mathrm{M} / \mathrm{D} / \mathrm{Y})$ & Gas (MMScf) & FWHP (psig) & Water (Bbl) \\
\hline \multicolumn{4}{|l|}{ January 2007} \\
\hline $1 / 1 / 2007$ & 23.004 & 1575 & 4.038 \\
\hline $1 / 2 / 2007$ & 25.402 & 1540 & 4.265 \\
\hline $1 / 3 / 2007$ & 30.842 & 1495 & 4.472 \\
\hline $1 / 4 / 2007$ & 34.922 & 1491 & 4.12 \\
\hline $1 / 5 / 2007$ & 40.858 & 1488 & 4.693 \\
\hline $1 / 6 / 2007$ & 41.619 & 1484 & 4.8 \\
\hline $1 / 7 / 2007$ & 42.466 & 1468 & 4.837 \\
\hline $1 / 8 / 2007$ & 42.754 & 1445 & 5.523 \\
\hline $1 / 9 / 2007$ & 43.349 & 1435 & 5.46 \\
\hline $1 / 10 / 2007$ & 43.147 & 1432 & 5.095 \\
\hline $1 / 11 / 2007$ & 43.435 & 1429 & 5.152 \\
\hline $1 / 12 / 2007$ & 43.369 & 1434 & 5.07 \\
\hline $1 / 13 / 2007$ & 43.470 & 1433 & 5.158 \\
\hline $1 / 14 / 2007$ & 43.215 & 1433 & 5.039 \\
\hline $1 / 15 / 2007$ & 43.298 & 1435 & 4.906 \\
\hline $1 / 16 / 2007$ & 43.221 & 1433 & 8.354 \\
\hline $1 / 17 / 2007$ & 43.237 & 1433 & 7.769 \\
\hline $1 / 18 / 2007$ & 43.154 & 1432 & 7.536 \\
\hline $1 / 19 / 2007$ & 43.211 & 1433 & 7.743 \\
\hline $1 / 20 / 2007$ & 43.212 & 1433 & 7.813 \\
\hline $1 / 21 / 2007$ & 43.343 & 1434 & 7.687 \\
\hline $1 / 22 / 2007$ & 43.231 & 1433 & 7.699 \\
\hline $1 / 23 / 2007$ & 43.293 & 1435 & 7.511 \\
\hline $1 / 24 / 2007$ & 43.168 & 1433 & 7.743 \\
\hline $1 / 25 / 2007$ & 43.168 & 1433 & 7.731 \\
\hline $1 / 26 / 2007$ & 43.241 & 1433 & 5.076 \\
\hline $1 / 27 / 2007$ & 43.252 & 1433 & 8.983 \\
\hline $1 / 28 / 2007$ & 43.215 & 1433 & 8.177 \\
\hline $1 / 29 / 2007$ & 43.235 & 1433 & 7.687 \\
\hline $1 / 30 / 2007$ & 43.234 & 1433 & 4.963 \\
\hline $1 / 31 / 2007$ & 43.214 & 1433 & 5.039 \\
\hline \multicolumn{4}{|l|}{ February 2007} \\
\hline $2 / 1 / 2007$ & 43.245 & 1440 & 5.73 \\
\hline $2 / 2 / 2007$ & 43.158 & 1438 & 5.819 \\
\hline $2 / 3 / 2007$ & 43.196 & 1439 & 7.53 \\
\hline $2 / 4 / 2007$ & 43.325 & 1442 & 6.787 \\
\hline $2 / 5 / 2007$ & 43.311 & 1442 & 6.649 \\
\hline $2 / 6 / 2007$ & 43.273 & 1442 & 5.844 \\
\hline $2 / 7 / 2007$ & 43.333 & 1441 & 5.988 \\
\hline 2/8/2007 & 43.360 & 1442 & 5.353 \\
\hline 2/9/2007 & 43.755 & 1440 & 5.51 \\
\hline
\end{tabular}

Table 6 continued

\begin{tabular}{|c|c|c|c|}
\hline Date $(\mathrm{M} / \mathrm{D} / \mathrm{Y})$ & Gas (MMScf) & FWHP (psig) & Water (Bbl) \\
\hline $2 / 10 / 2007$ & 43.701 & 1437 & 4.906 \\
\hline $2 / 11 / 2007$ & 43.798 & 1437 & 5.007 \\
\hline $2 / 12 / 2007$ & 43.723 & 1436 & 5.447 \\
\hline $2 / 13 / 2007$ & 43.786 & 1438 & 5.988 \\
\hline $2 / 14 / 2007$ & 43.809 & 1438 & 5.881 \\
\hline $2 / 15 / 2007$ & 43.783 & 1438 & 5.083 \\
\hline $2 / 16 / 2007$ & 43.788 & 1437 & 4.938 \\
\hline $2 / 17 / 2007$ & 43.783 & 1437 & 5.391 \\
\hline $2 / 18 / 2007$ & 43.776 & 1436 & 4.875 \\
\hline $2 / 19 / 2007$ & 43.743 & 1436 & 6.605 \\
\hline $2 / 20 / 2007$ & 43.886 & 1437 & 5.215 \\
\hline $2 / 21 / 2007$ & 43.769 & 1437 & 5.397 \\
\hline $2 / 22 / 2007$ & 43.797 & 1436 & 5.271 \\
\hline $2 / 23 / 2007$ & 43.801 & 1437 & 8.146 \\
\hline $2 / 24 / 2007$ & 43.704 & 1436 & 6.611 \\
\hline $2 / 25 / 2007$ & 43.705 & 1436 & 6.284 \\
\hline $2 / 26 / 2007$ & 43.798 & 1437 & 6.108 \\
\hline $2 / 27 / 2007$ & 43.784 & 1437 & 6.209 \\
\hline $2 / 28 / 2007$ & 43.746 & 1437 & 5.309 \\
\hline \multicolumn{4}{|l|}{ March 2007} \\
\hline $3 / 1 / 2007$ & 43.734 & 1433 & 6.058 \\
\hline $3 / 2 / 2007$ & 43.33 & 1432 & 5.328 \\
\hline $3 / 3 / 2007$ & 43.744 & 1430 & 6.076 \\
\hline $3 / 4 / 2007$ & 43.719 & 1428 & 5.756 \\
\hline $3 / 5 / 2007$ & 43.827 & 1428 & 5.806 \\
\hline $3 / 6 / 2007$ & 43.748 & 1429 & 5.674 \\
\hline $3 / 7 / 2007$ & 43.742 & 1429 & 5.743 \\
\hline $3 / 8 / 2007$ & 43.804 & 1428 & 5.611 \\
\hline $3 / 9 / 2007$ & 42.926 & 1437 & 5.504 \\
\hline $3 / 10 / 2007$ & 43.815 & 1429 & 5.24 \\
\hline $3 / 11 / 2007$ & 43.778 & 1429 & 5.101 \\
\hline $3 / 12 / 2007$ & 43.853 & 1429 & 5.793 \\
\hline $3 / 13 / 2007$ & 43.829 & 1429 & 5.624 \\
\hline $3 / 14 / 2007$ & 43.872 & 1429 & 5.756 \\
\hline $3 / 15 / 2007$ & 43.862 & 1428 & 5.385 \\
\hline $3 / 16 / 2007$ & 43.252 & 1431 & 3.548 \\
\hline $3 / 17 / 2007$ & 43.355 & 1430 & 6.504 \\
\hline $3 / 18 / 2007$ & 43.646 & 1431 & 5.598 \\
\hline $3 / 19 / 2007$ & 43.905 & 1433 & 5.617 \\
\hline $3 / 20 / 2007$ & 42.348 & 1439 & 7.725 \\
\hline $3 / 21 / 2007$ & 41.008 & 1445 & 5.699 \\
\hline $3 / 22 / 2007$ & 40.248 & 1452 & 7.926 \\
\hline $3 / 23 / 2007$ & 40.108 & 1455 & 7.536 \\
\hline $3 / 24 / 2007$ & 40.505 & 1450 & 5.296 \\
\hline $3 / 25 / 2007$ & 40.136 & 1452 & 4.63 \\
\hline $3 / 26 / 2007$ & 39.723 & 1455 & 4.711 \\
\hline $3 / 27 / 2007$ & 40.31 & 1450 & 5.083 \\
\hline $3 / 28 / 2007$ & 40.392 & 1449 & 7.97 \\
\hline
\end{tabular}


Table 6 continued

\begin{tabular}{|c|c|c|c|}
\hline Date $(\mathrm{M} / \mathrm{D} / \mathrm{Y})$ & Gas (MMScf) & FWHP (psig) & Water (Bbl) \\
\hline $3 / 29 / 2007$ & 40.312 & 1450 & 4.554 \\
\hline $3 / 30 / 2007$ & 40.371 & 1449 & 4.642 \\
\hline $3 / 31 / 2007$ & 40.436 & 1449 & 6.064 \\
\hline \multicolumn{4}{|l|}{ April 2007} \\
\hline $4 / 1 / 2007$ & 40.241 & 1486 & 4.498 \\
\hline $4 / 2 / 2007$ & 40.466 & 1490 & 4.592 \\
\hline $4 / 3 / 2007$ & 40.281 & 1490 & 4.749 \\
\hline $4 / 4 / 2007$ & 40.338 & 1489 & 4.485 \\
\hline $4 / 5 / 2007$ & 40.332 & 1491 & 4.441 \\
\hline $4 / 6 / 2007$ & 40.298 & 1490 & 5.397 \\
\hline $4 / 7 / 2007$ & 39.81 & 1495 & 4.724 \\
\hline $4 / 8 / 2007$ & 40.698 & 1488 & 4.233 \\
\hline 4/9/2007 & 39.626 & 1497 & 4.925 \\
\hline $4 / 10 / 2007$ & 39.961 & 1496 & 3.585 \\
\hline $4 / 11 / 2007$ & 40.403 & 1491 & 3.843 \\
\hline $4 / 12 / 2007$ & 40.4 & 1489 & 4.347 \\
\hline $4 / 13 / 2007$ & 40.162 & 1493 & 1.963 \\
\hline $4 / 14 / 2007$ & 40.096 & 1492 & 4.328 \\
\hline $4 / 15 / 2007$ & 40.275 & 1492 & 4.24 \\
\hline $4 / 16 / 2007$ & 39.972 & 1493 & 4.221 \\
\hline $4 / 17 / 2007$ & 40.274 & 1492 & 3.825 \\
\hline $4 / 18 / 2007$ & 39.678 & 1498 & 6.919 \\
\hline $4 / 19 / 2007$ & 39.717 & 1496 & 4.542 \\
\hline $4 / 20 / 2007$ & 39.796 & 1495 & 4.661 \\
\hline $4 / 21 / 2007$ & 40.069 & 1493 & 4.227 \\
\hline $4 / 22 / 2007$ & 39.924 & 1496 & 4.636 \\
\hline $4 / 23 / 2007$ & 39.708 & 1497 & 4.397 \\
\hline $4 / 24 / 2007$ & 40.151 & 1494 & 4.623 \\
\hline $4 / 25 / 2007$ & 40.176 & 1494 & 4.9 \\
\hline $4 / 26 / 2007$ & 40.205 & 1493 & 4.825 \\
\hline $4 / 27 / 2007$ & 39.736 & 1495 & 4.523 \\
\hline $4 / 28 / 2007$ & 40.228 & 1491 & 4.743 \\
\hline $4 / 29 / 2007$ & 40.272 & 1491 & 4.661 \\
\hline $4 / 30 / 2007$ & 39.846 & 1495 & 4.598 \\
\hline \multicolumn{4}{|l|}{ May 2007} \\
\hline $5 / 1 / 2007$ & 40.056 & 1485 & 4.37784 \\
\hline $5 / 2 / 2007$ & 40.267 & 1481 & 4.34639 \\
\hline $5 / 3 / 2007$ & 40.401 & 1478 & 4.92507 \\
\hline $5 / 4 / 2007$ & 40.139 & 1478 & 4.45332 \\
\hline $5 / 5 / 2007$ & 40.158 & 1481 & 4.68605 \\
\hline $5 / 6 / 2007$ & 40.334 & 1477 & 4.86217 \\
\hline $5 / 7 / 2007$ & 40.066 & 1479 & 4.47848 \\
\hline $5 / 8 / 2007$ & 40.271 & 1479 & 4.57912 \\
\hline $5 / 9 / 2007$ & 40.215 & 1480 & 4.73008 \\
\hline $5 / 10 / 2007$ & 40.053 & 1482 & 4.62315 \\
\hline $5 / 11 / 2007$ & 40.056 & 1481 & 4.66089 \\
\hline $5 / 12 / 2007$ & 40.113 & 1479 & 4.7804 \\
\hline $5 / 13 / 2007$ & 40.286 & 1478 & 4.56025 \\
\hline
\end{tabular}

Table 6 continued

\begin{tabular}{|c|c|c|c|}
\hline Date $(\mathrm{M} / \mathrm{D} / \mathrm{Y})$ & Gas (MMScf) & FWHP (psig) & Water (Bbl) \\
\hline $5 / 14 / 2007$ & 40.310 & 1479 & 4.52251 \\
\hline $5 / 15 / 2007$ & 40.281 & 1480 & 4.62315 \\
\hline $5 / 16 / 2007$ & 40.293 & 1478 & 4.58541 \\
\hline $5 / 17 / 2007$ & 40.185 & 1479 & 4.4659 \\
\hline $5 / 18 / 2007$ & 40.219 & 1479 & 4.74895 \\
\hline $5 / 19 / 2007$ & 40.376 & 1478 & 4.30236 \\
\hline $5 / 20 / 2007$ & 40.278 & 1480 & 4.62315 \\
\hline $5 / 21 / 2007$ & 40.041 & 1480 & 4.60428 \\
\hline $5 / 22 / 2007$ & 40.282 & 1478 & 4.76782 \\
\hline $5 / 23 / 2007$ & 40.041 & 1481 & 4.57912 \\
\hline $5 / 24 / 2007$ & 40.331 & 1477 & 4.69234 \\
\hline $5 / 25 / 2007$ & 40.246 & 1477 & 4.49735 \\
\hline $5 / 26 / 2007$ & 40.240 & 1479 & 4.70492 \\
\hline $5 / 27 / 2007$ & 40.105 & 1480 & 4.67976 \\
\hline $5 / 28 / 2007$ & 40.231 & 1477 & 4.67976 \\
\hline $5 / 29 / 2007$ & 40.084 & 1482 & 4.49106 \\
\hline $5 / 30 / 2007$ & 39.996 & 1481 & 4.67347 \\
\hline $5 / 31 / 2007$ & 40.534 & 1478 & 3.69852 \\
\hline \multicolumn{4}{|l|}{ June 2007} \\
\hline $6 / 1 / 2007$ & 40.166 & 1485 & 4.4659 \\
\hline $6 / 2 / 2007$ & 40.204 & 1487 & 4.59799 \\
\hline $6 / 3 / 2007$ & 40.094 & 1490 & 4.56025 \\
\hline $6 / 4 / 2007$ & 40.251 & 1488 & 4.81185 \\
\hline $6 / 5 / 2007$ & 40.278 & 1488 & 4.56025 \\
\hline $6 / 6 / 2007$ & 40.121 & 1489 & 4.52251 \\
\hline 6/7/2007 & 40.128 & 1488 & 4.5288 \\
\hline 6/8/2007 & 40.387 & 1485 & 4.7175 \\
\hline 6/9/2007 & 40.293 & 1486 & 4.76782 \\
\hline $6 / 10 / 2007$ & 40.248 & 1487 & 4.66089 \\
\hline $6 / 11 / 2007$ & 40.331 & 1488 & 4.7804 \\
\hline $6 / 12 / 2007$ & 40.013 & 1489 & 4.55396 \\
\hline $6 / 13 / 2007$ & 40.244 & 1488 & 4.49106 \\
\hline $6 / 14 / 2007$ & 40.245 & 1487 & 4.57283 \\
\hline $6 / 15 / 2007$ & 40.229 & 1486 & 4.69234 \\
\hline $6 / 16 / 2007$ & 40.383 & 1486 & 4.78669 \\
\hline $6 / 17 / 2007$ & 40.392 & 1484 & 3.96899 \\
\hline $6 / 18 / 2007$ & 40.322 & 1488 & 3.92496 \\
\hline $6 / 19 / 2007$ & 40.478 & 1487 & 4.5288 \\
\hline $6 / 20 / 2007$ & 40.640 & 1485 & 4.76782 \\
\hline $6 / 21 / 2007$ & 40.455 & 1487 & 3.95012 \\
\hline $6 / 22 / 2007$ & 40.341 & 1486 & 4.51622 \\
\hline $6 / 23 / 2007$ & 40.298 & 1488 & 4.37784 \\
\hline $6 / 24 / 2007$ & 40.370 & 1487 & 4.30865 \\
\hline $6 / 25 / 2007$ & 40.513 & 1485 & 4.52251 \\
\hline $6 / 26 / 2007$ & 40.462 & 1486 & 4.30865 \\
\hline $6 / 27 / 2007$ & 40.414 & 1487 & 4.81185 \\
\hline $6 / 28 / 2007$ & 40.093 & 1489 & 4.77411 \\
\hline $6 / 29 / 2007$ & 40.324 & 1487 & 4.78669 \\
\hline
\end{tabular}


Table 6 continued

\begin{tabular}{|c|c|c|c|}
\hline Date $(\mathrm{M} / \mathrm{D} / \mathrm{Y})$ & Gas (MMScf) & FWHP (psig) & Water (Bbl) \\
\hline $6 / 30 / 2007$ & 40.191 & 1488 & 4.74895 \\
\hline \multicolumn{4}{|l|}{ July 2007} \\
\hline $7 / 1 / 2007$ & 40.154 & 1486 & 4.66089 \\
\hline $7 / 2 / 2007$ & 40.329 & 1481 & 4.56654 \\
\hline $7 / 3 / 2007$ & 40.337 & 1477 & 4.64202 \\
\hline $7 / 4 / 2007$ & 40.375 & 1477 & 4.54138 \\
\hline $7 / 5 / 2007$ & 40.353 & 1477 & 4.58541 \\
\hline $7 / 6 / 2007$ & 40.295 & 1478 & 4.64831 \\
\hline $7 / 7 / 2007$ & 40.342 & 1478 & 4.64831 \\
\hline $7 / 8 / 2007$ & 40.352 & 1477 & 4.82443 \\
\hline 7/9/2007 & 40.324 & 1475 & 4.66718 \\
\hline $7 / 10 / 2007$ & 40.463 & 1477 & 4.62944 \\
\hline $7 / 11 / 2007$ & 40.458 & 1477 & 4.59799 \\
\hline $7 / 12 / 2007$ & 40.566 & 1478 & 4.5917 \\
\hline $7 / 13 / 2007$ & 40.412 & 1476 & 4.24575 \\
\hline $7 / 14 / 2007$ & 40.543 & 1477 & 4.49735 \\
\hline $7 / 15 / 2007$ & 40.453 & 1476 & 4.74895 \\
\hline $7 / 16 / 2007$ & 40.132 & 1479 & 4.74895 \\
\hline $7 / 17 / 2007$ & 40.419 & 1477 & 4.68605 \\
\hline $7 / 18 / 2007$ & 40.087 & 1481 & 4.71121 \\
\hline $7 / 19 / 2007$ & 40.117 & 1480 & 4.69234 \\
\hline $7 / 20 / 2007$ & 40.105 & 1481 & 4.64202 \\
\hline $7 / 21 / 2007$ & 40.164 & 1479 & 4.47219 \\
\hline $7 / 22 / 2007$ & 40.334 & 1476 & 4.54138 \\
\hline $7 / 23 / 2007$ & 39.840 & 1482 & 4.58541 \\
\hline $7 / 24 / 2007$ & 39.921 & 1480 & 4.59799 \\
\hline $7 / 25 / 2007$ & 39.694 & 1485 & 4.04447 \\
\hline $7 / 26 / 2007$ & 40.291 & 1479 & 4.74895 \\
\hline $7 / 27 / 2007$ & 40.130 & 1480 & 4.7175 \\
\hline $7 / 28 / 2007$ & 40.035 & 1481 & 4.50364 \\
\hline $7 / 29 / 2007$ & 39.953 & 1481 & 4.61686 \\
\hline $7 / 30 / 2007$ & 40.262 & 1478 & 4.49735 \\
\hline $7 / 31 / 2007$ & 40.239 & 1479 & 4.5917 \\
\hline \multicolumn{4}{|l|}{ August 2007} \\
\hline $8 / 1 / 2007$ & 39.945 & 1477 & 4.40929 \\
\hline $8 / 2 / 2007$ & 40.235 & 1474 & 4.50993 \\
\hline $8 / 3 / 2007$ & 0.832 & 1675 & 0.73593 \\
\hline $8 / 4 / 2007$ & 0.000 & 1677 & 0.00 \\
\hline $8 / 5 / 2007$ & 26.272 & 1580 & 3.04436 \\
\hline $8 / 6 / 2007$ & 39.846 & 1479 & 4.7804 \\
\hline $8 / 7 / 2007$ & 40.038 & 1478 & 4.57283 \\
\hline $8 / 8 / 2007$ & 39.955 & 1480 & 4.37155 \\
\hline $8 / 9 / 2007$ & 39.875 & 1479 & 4.70492 \\
\hline $8 / 10 / 2007$ & 40.094 & 1478 & 4.13253 \\
\hline $8 / 11 / 2007$ & 40.190 & 1476 & 4.28349 \\
\hline $8 / 12 / 2007$ & 40.023 & 1478 & 4.61057 \\
\hline $8 / 13 / 2007$ & 40.224 & 1475 & 4.73008 \\
\hline $8 / 14 / 2007$ & 39.832 & 1481 & 4.42816 \\
\hline
\end{tabular}

Table 6 continued

\begin{tabular}{|c|c|c|c|}
\hline Date (M/D/Y) & Gas (MMScf) & FWHP (psig) & Water (Bbl) \\
\hline $8 / 15 / 2007$ & 40.044 & 1476 & 4.55396 \\
\hline $8 / 16 / 2007$ & 40.109 & 1475 & 4.67976 \\
\hline $8 / 17 / 2007$ & 39.958 & 1478 & 4.73008 \\
\hline $8 / 18 / 2007$ & 40.010 & 1478 & 4.86217 \\
\hline $8 / 19 / 2007$ & 40.190 & 1476 & 4.69234 \\
\hline $8 / 20 / 2007$ & 40.296 & 1475 & 4.60428 \\
\hline $8 / 21 / 2007$ & 40.423 & 1473 & 4.74895 \\
\hline $8 / 22 / 2007$ & 40.042 & 1478 & 4.56025 \\
\hline $8 / 23 / 2007$ & 39.885 & 1481 & 4.39042 \\
\hline $8 / 24 / 2007$ & 40.083 & 1476 & 4.66089 \\
\hline $8 / 25 / 2007$ & 40.079 & 1477 & 4.64831 \\
\hline $8 / 26 / 2007$ & 40.206 & 1476 & 4.67347 \\
\hline $8 / 27 / 2007$ & 39.977 & 1478 & 4.50993 \\
\hline $8 / 28 / 2007$ & 39.958 & 1478 & 4.67976 \\
\hline $8 / 29 / 2007$ & 40.421 & 1475 & 4.03818 \\
\hline $8 / 30 / 2007$ & 40.324 & 1474 & 4.64202 \\
\hline $8 / 31 / 2007$ & 40.539 & 1472 & 4.64831 \\
\hline \multicolumn{4}{|l|}{ September 2007} \\
\hline $9 / 1 / 2007$ & 40.262 & 1480 & 4.61686 \\
\hline $9 / 2 / 2007$ & 40.159 & 1485 & 4.62944 \\
\hline $9 / 3 / 2007$ & 40.267 & 1483 & 4.86217 \\
\hline 9/4/2007 & 39.895 & 1489 & 4.7175 \\
\hline $9 / 5 / 2007$ & 40.457 & 1484 & 4.77411 \\
\hline 9/6/2007 & 40.171 & 1486 & 4.71121 \\
\hline 9/7/2007 & 40.040 & 1489 & 4.76153 \\
\hline $9 / 8 / 2007$ & 40.405 & 1484 & 5.55407 \\
\hline 9/9/2007 & 40.270 & 1485 & 4.80556 \\
\hline 9/10/2007 & 40.221 & 1486 & 4.48477 \\
\hline $9 / 11 / 2007$ & 40.331 & 1483 & 4.78669 \\
\hline $9 / 12 / 2007$ & 40.509 & 1484 & 4.66718 \\
\hline $9 / 13 / 2007$ & 40.469 & 1485 & 4.63573 \\
\hline $9 / 14 / 2007$ & 40.483 & 1482 & 4.66718 \\
\hline $9 / 15 / 2007$ & 40.425 & 1483 & 4.66718 \\
\hline $9 / 16 / 2007$ & 40.243 & 1485 & 4.50993 \\
\hline 9/17/2007 & 40.512 & 1484 & 9.46016 \\
\hline $9 / 18 / 2007$ & 40.538 & 1482 & 8.98841 \\
\hline $9 / 19 / 2007$ & 40.081 & 1484 & 7.51026 \\
\hline $9 / 20 / 2007$ & 40.062 & 1485 & 4.81185 \\
\hline $9 / 21 / 2007$ & 40.566 & 1483 & 5.67358 \\
\hline $9 / 22 / 2007$ & 40.180 & 1485 & 3.82432 \\
\hline $9 / 23 / 2007$ & 40.051 & 1489 & 4.64202 \\
\hline $9 / 24 / 2007$ & 40.502 & 1484 & 4.91249 \\
\hline $9 / 25 / 2007$ & 40.130 & 1485 & 4.72379 \\
\hline $9 / 26 / 2007$ & 40.366 & 1484 & 4.70492 \\
\hline $9 / 27 / 2007$ & 40.229 & 1485 & 4.79298 \\
\hline $9 / 28 / 2007$ & 40.388 & 1484 & 4.88733 \\
\hline $9 / 29 / 2007$ & 40.133 & 1486 & 4.13253 \\
\hline $9 / 30 / 2007$ & 40.367 & 1485 & 4.42187 \\
\hline
\end{tabular}


Table 6 continued

\begin{tabular}{|c|c|c|c|c|c|c|c|}
\hline Date $(\mathrm{M} / \mathrm{D} / \mathrm{Y})$ & Gas (MMScf) & FWHP (psig) & Water (Bbl) & Date $(\mathrm{M} / \mathrm{D} / \mathrm{Y})$ & Gas (MMScf) & FWHP (psig) & Water (Bbl) \\
\hline October 2007 & & & & $11 / 16 / 2007$ & 22.321 & 1505 & 2.91856 \\
\hline $10 / 1 / 2007$ & 40.855 & 1483 & 4.01931 & $11 / 17 / 2007$ & 39.769 & 1481 & 4.41558 \\
\hline $10 / 2 / 2007$ & 40.438 & 1482 & 4.73008 & $11 / 18 / 2007$ & 40.361 & 1475 & 4.54138 \\
\hline $10 / 3 / 2007$ & 40.227 & 1484 & 4.82443 & $11 / 19 / 2007$ & 40.252 & 1475 & 4.66089 \\
\hline $10 / 4 / 2007$ & 40.439 & 1485 & 4.56654 & $11 / 20 / 2007$ & 40.187 & 1474 & 4.88104 \\
\hline $10 / 5 / 2007$ & 40.102 & 1486 & 4.89991 & $11 / 21 / 2007$ & 40.290 & 1475 & 4.60428 \\
\hline $10 / 6 / 2007$ & 40.066 & 1488 & 4.98168 & $11 / 22 / 2007$ & 40.198 & 1475 & 4.58541 \\
\hline $10 / 7 / 2007$ & 40.563 & 1485 & 4.45961 & $11 / 23 / 2007$ & 40.169 & 1476 & 4.45961 \\
\hline $10 / 8 / 2007$ & 40.343 & 1485 & 4.66718 & $11 / 24 / 2007$ & 40.422 & 1473 & 4.69863 \\
\hline $10 / 9 / 2007$ & 40.459 & 1484 & 4.32752 & $11 / 25 / 2007$ & 40.189 & 1477 & 4.57912 \\
\hline $10 / 10 / 2007$ & 39.981 & 1488 & 4.54767 & $11 / 26 / 2007$ & 40.156 & 1477 & 4.5917 \\
\hline $10 / 11 / 2007$ & 39.914 & 1489 & 3.9627 & $11 / 27 / 2007$ & 40.069 & 1476 & 4.74266 \\
\hline $10 / 12 / 2007$ & 39.825 & 1490 & 4.41558 & $11 / 28 / 2007$ & 40.234 & 1474 & 4.79927 \\
\hline $10 / 13 / 2007$ & 36.469 & 1525 & 4.20172 & $11 / 29 / 2007$ & 40.194 & 1477 & 4.69234 \\
\hline $10 / 14 / 2007$ & 36.504 & 1525 & 4.11995 & $11 / 30 / 2007$ & 40.263 & 1475 & 4.57912 \\
\hline $10 / 15 / 2007$ & 33.905 & 1540 & 3.97528 & December 2007 & & & \\
\hline $10 / 16 / 2007$ & 34.814 & 1536 & 4.10737 & $12 / 1 / 2007$ & 40.441 & 1473 & 4.58541 \\
\hline $10 / 17 / 2007$ & 39.415 & 1494 & 4.52251 & $12 / 2 / 2007$ & 40.316 & 1470 & 4.75524 \\
\hline $10 / 18 / 2007$ & 40.171 & 1487 & 4.23317 & $12 / 3 / 2007$ & 40.398 & 1469 & 4.18285 \\
\hline $10 / 19 / 2007$ & 39.917 & 1489 & 4.64202 & $12 / 4 / 2007$ & 40.380 & 1468 & 4.30236 \\
\hline $10 / 20 / 2007$ & 39.974 & 1489 & 4.66089 & $12 / 5 / 2007$ & 39.977 & 1474 & 4.42187 \\
\hline $10 / 21 / 2007$ & 40.001 & 1488 & 4.74266 & $12 / 6 / 2007$ & 39.892 & 1474 & 4.66718 \\
\hline $10 / 22 / 2007$ & 40.126 & 1487 & 4.06334 & $12 / 7 / 2007$ & 40.004 & 1473 & 4.59799 \\
\hline $10 / 23 / 2007$ & 39.999 & 1490 & 4.69863 & $12 / 8 / 2007$ & 39.379 & 1478 & 4.47848 \\
\hline $10 / 24 / 2007$ & 39.940 & 1487 & 4.66089 & $12 / 9 / 2007$ & 39.807 & 1475 & 4.61686 \\
\hline $10 / 25 / 2007$ & 39.822 & 1490 & 4.4659 & $12 / 10 / 2007$ & 39.580 & 1476 & 4.5288 \\
\hline $10 / 26 / 2007$ & 40.027 & 1488 & 4.41558 & $12 / 11 / 2007$ & 39.783 & 1474 & 4.60428 \\
\hline $10 / 27 / 2007$ & 40.213 & 1486 & 4.68605 & $12 / 12 / 2007$ & 40.164 & 1471 & 4.62315 \\
\hline $10 / 28 / 2007$ & 40.060 & 1486 & 4.77411 & $12 / 13 / 2007$ & 39.997 & 1472 & 4.42816 \\
\hline $10 / 29 / 2007$ & 40.081 & 1489 & 4.56025 & $12 / 14 / 2007$ & 40.424 & 1467 & 4.81185 \\
\hline $10 / 30 / 2007$ & 39.939 & 1489 & 4.74895 & $12 / 15 / 2007$ & 40.232 & 1468 & 4.56654 \\
\hline $10 / 31 / 2007$ & 40.399 & 1484 & 4.81185 & $12 / 16 / 2007$ & 40.015 & 1473 & 4.43445 \\
\hline November 2007 & & & & $12 / 17 / 2007$ & 40.372 & 1468 & 4.47219 \\
\hline $11 / 1 / 2007$ & 40.160 & 1477 & 4.64831 & $12 / 18 / 2007$ & 40.364 & 1470 & 4.52251 \\
\hline $11 / 2 / 2007$ & 40.205 & 1475 & 4.48477 & $12 / 19 / 2007$ & 40.342 & 1467 & 4.54138 \\
\hline $11 / 3 / 2007$ & 40.093 & 1477 & 4.70492 & $12 / 20 / 2007$ & 36.723 & 1495 & 3.61046 \\
\hline $11 / 4 / 2007$ & 39.964 & 1478 & 4.50364 & $12 / 21 / 2007$ & 38.039 & 1492 & 3.76771 \\
\hline $11 / 5 / 2007$ & 40.127 & 1477 & 4.4659 & $12 / 22 / 2007$ & 38.698 & 1491 & 4.12624 \\
\hline $11 / 6 / 2007$ & 39.986 & 1477 & 4.57283 & $12 / 23 / 2007$ & 38.891 & 1490 & 4.42187 \\
\hline $11 / 7 / 2007$ & 40.022 & 1476 & 4.70492 & $12 / 24 / 2007$ & 39.896 & 1477 & 4.20172 \\
\hline $11 / 8 / 2007$ & 39.947 & 1479 & 4.44703 & $12 / 25 / 2007$ & 39.896 & 1473 & 5.86228 \\
\hline $11 / 9 / 2007$ & 40.050 & 1477 & 4.62944 & $12 / 26 / 2007$ & 39.164 & 1482 & 4.41558 \\
\hline $11 / 10 / 2007$ & 40.138 & 1474 & 4.44074 & $12 / 27 / 2007$ & 40.044 & 1472 & 4.8433 \\
\hline $11 / 11 / 2007$ & 40.074 & 1479 & 4.39671 & $12 / 28 / 2007$ & 39.872 & 1476 & 4.44074 \\
\hline $11 / 12 / 2007$ & 39.945 & 1477 & 4.49106 & $12 / 29 / 2007$ & 39.675 & 1478 & 4.60428 \\
\hline $11 / 13 / 2007$ & 39.865 & 1479 & 4.45961 & $12 / 30 / 2007$ & 36.419 & 1497 & 4.24575 \\
\hline $11 / 14 / 2007$ & 40.139 & 1474 & 4.69234 & $12 / 31 / 2007$ & 40.064 & 1473 & 4.6546 \\
\hline
\end{tabular}

Table 6 continued 


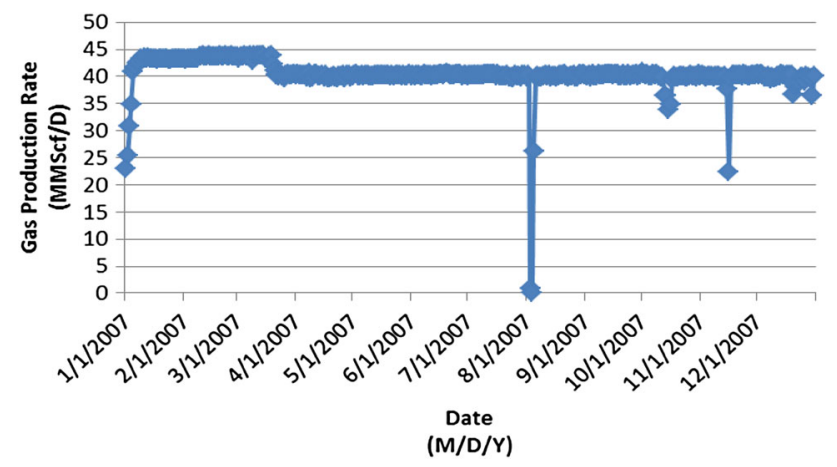

Fig. 13 Graphical presentation of gas production rate of Well-10 during the year of 2007

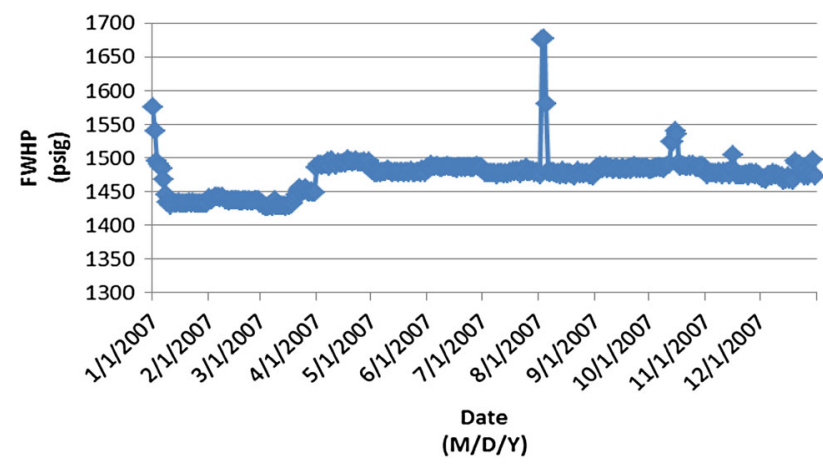

Fig. 14 Graphical presentation of flowing wellhead pressure (FWHP) of Well-10 during the year of 2007

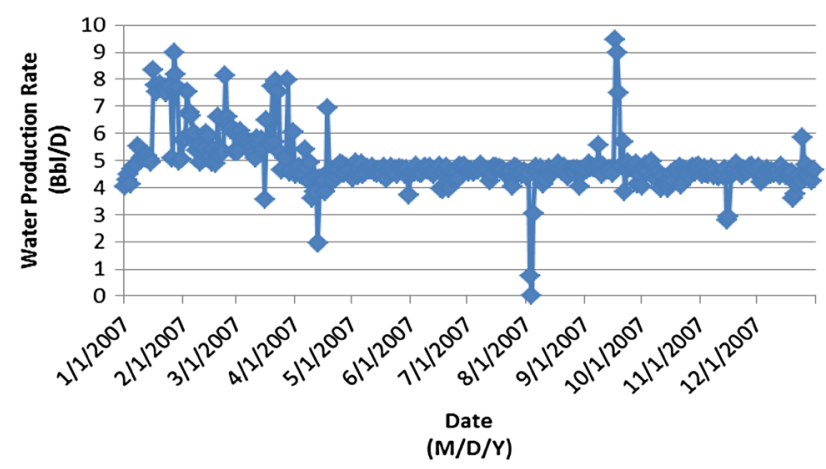

Fig. 15 Graphical presentation of water production rate of Well-10 during the year of 2007

\section{References}

Altered zone and skin effect (2016) Petrowiki. http://petrowiki.org/ Fluid_flow_with_formation_damage. Accessed 1 June 2016

Bangladesh Gas Fields Company Limited (BGFCL) (2014). http:// www.bgfcl.org.bd/habiganj_map.html. Accessed July 52014
Clarkson CR (2013) Production data analysis of unconventional gas wells: review of theory and best practices. Int $\mathrm{J}$ Coal Geol 109-110:101-146. doi:10.1016/j.coal.2013.01.002

Clarkson CR, Jensen JL, Blasingame TA (2012) Unconventional gas reservoir evaluation: what do we have to consider? Journal of Natural Gas Science \& Engineering 8(9):9-33. doi:10.1016/j. jngse.2012.01.001

Fetkovich MJ (1980) Decline curve analysis using type curves. J Petrol Technol. doi:10.2118/4629-PA

Fetkovich MJ, Vienot ME, Bradley MD, Kiesow UG (1987) Decline curve analysis using type curves: case histories. SPE Formation Evaluation. doi:10.2118/13169-PAb

Fetkovich MJ, Fetkovich EJ, Fetkovich MD (1996) Useful concepts for decline curve forecasting, reserve estimation, and analysis. SPE Reservoir Engineering. doi:10.2118/28628-PA

Gringarten AC (1987) Type-curve analysis: what it can and cannot do. J Petrol Technol 39(01):11-13. doi:10.2118/16388-PA

Haq MB, Gomes E (2001) Estimation of gas in place of Bangladesh using flowing material balance method. In: 4th international conference on mechanical engineering, Dhaka, Bangladesh, pp VII 83-88

Hartmann DJ, Beaumont EA (2016) Predicting reservoir system quality and performance. In: Exploring for oil and gas traps. AAPG Store. http://wiki.aapg.org/Reservoir_drive_mechanisms. Accessed 20 May 2016

Hawkins MF Jr (1956) A note on the skin effect. J Petrol Technol 8(12):65-66. doi:10.2118/732-G

Help Manual (2010) Software FEKETE, F.A.S.T.RTA ${ }^{\mathrm{TM}}$, version 4.5.1.277, IHS Inc

Imam B (2005) Energy resources of Bangladesh. University Grant Commission, Dhaka, p 135

Imam B (2013) Energy resources of Bangladesh, 2nd edn. University Grant Commission, Dhaka

Jensen JL, Hinkley DV, Lake LW (1987) A statistical study of reservoir permeability: distributions, correlations, and averages. SPE Formation Evaluation 2(4):461-468. doi:10.2118/14270-PA

Jianchun G, Cong L, Yong X, Jichuan R, Chaoyi S, Yu S (2014) Reservoir stimulation techniques to minimize skin factor of Longwangmiao Fm gas reservoirs in the Sichuan Basin. Natural Gas Industry B 1(1):83-88. doi:10.1016/j.ngib.2014.10.011

Ley HC, Samaniego F V (1981) Transient pressure analysis for fractured wells. J Petrol Technol 33(09):1749-1767. doi:10. 2118/7490-PA

Mireault R, Dean L (2007-2008) Reservoir engineering for geologists. Canadian society of petroleum geologists reservoir magazine, Calgary, Albert

Mishra S (2014) Exploring the diagnostic capability of RTA typecurves. In: SPE annual technical conference and exhibition, Amsterdam, Netherland. doi:10.2118/173481-STU

Morehouse DF (1997) The intricate puzzle of oil and gas reserve growth. In: Natural gas monthly (energy information administration). Accessed 8 Feb 2012

Van Everdingen AF (1953) The skin effect and its influence on the productive capacity of a well. J Petrol Technol. doi:10.2118/203G

Zolotukhin AB, Ursin JR (2000) Introduction to petroleum reservoir engineering. In: Høyskoleforlaget. Norwegian Academic Press, Kristiansand, Norway 